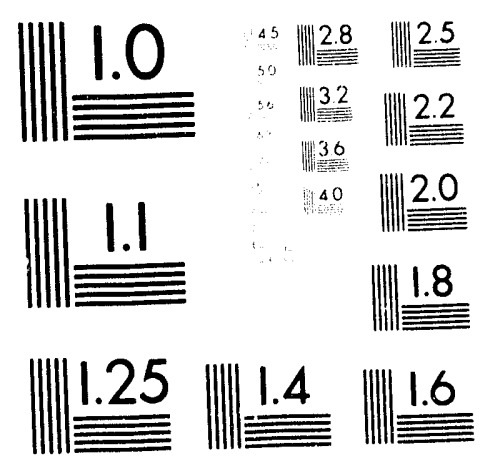



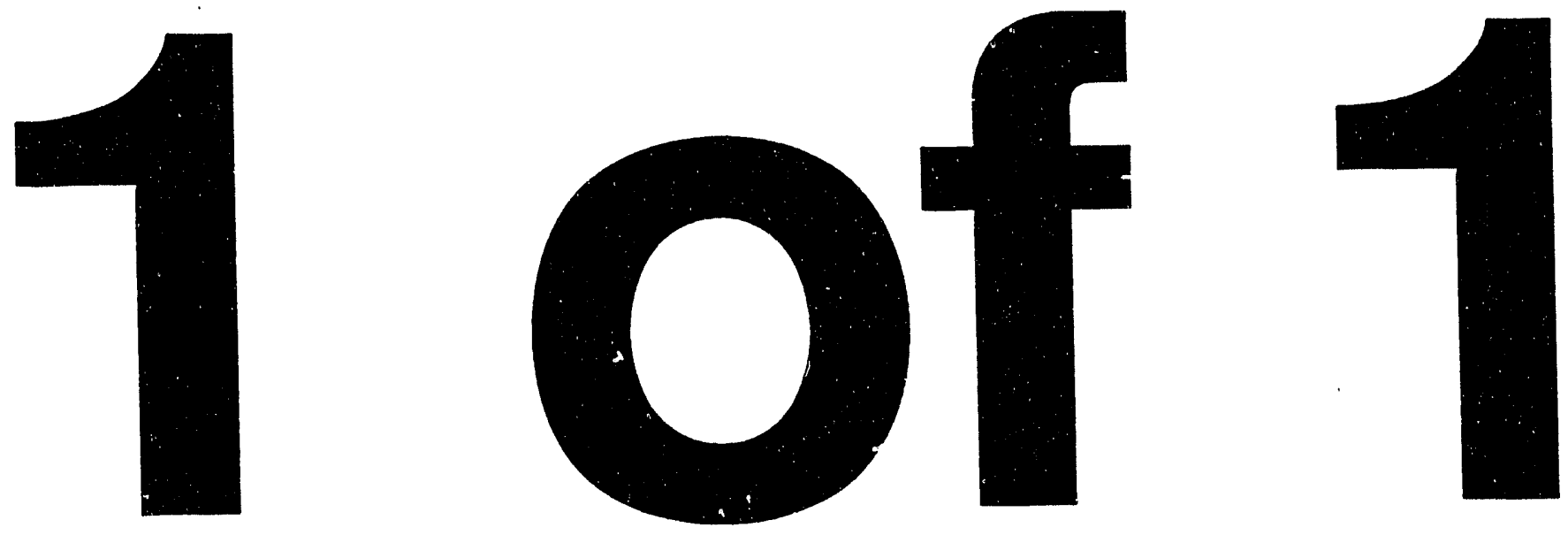
Engineering Physics arid Mathematics Division

Mathematical Sciences Section

\title{
THE DESIGN OF LINEAR ALGEBRA LIBRARIES FOR HIGH PERFORMANCE COMPUTERS
}

\author{
Jack J. Dongarra $\ddagger \S$ \\ David W. Walker $\S$ \\ $\ddagger$ Department of Computer Science \\ University of Tennessee \\ 107 Ayres Hall \\ Knoxville, TN 37996-1301 \\ $\S$ Mathematical Sciences Section \\ Oak Ridge National Laboratory \\ P.O. Box 2008, Bldg. 6012 \\ Oak Ridge, TN 37831-6367
}

Date Published: August 1993

Research was supported by the Applied Mathematical Sciences Research Program of the Office of Energy Research, U.S. Department of Energy, and by the Advanced Research Projects Agency under contract DAAL03-91-C-0047, administered by the Army Research Office.

\author{
Prepared by the \\ Oak Ridge National Laboratory \\ Oak Ridge, Tennessee 37831 \\ managed by \\ Martin Marietta Energy Systems, Inc. \\ for the \\ U.S. DEPARTMENT OF ENERGY \\ under Contract No. DE-AC05-84OR21400




\section{Contents}

1 Introduction . . . . . . . . . . . . . . . . . . . . . . . . 1

1.1 Dense Linear Algebra Libraries . . . . . . . . . . . . . . . . . . . . . . . . 2

1.1 .1 EISPACK . . . . . . . . . . . . . . . . . . . . 2

$1.1 .2 \quad$ LINPACK $\ldots \ldots \ldots \ldots \ldots$

1.1 .3 LAPACK . . . . . . . . . . . . . . . . . . . . 4

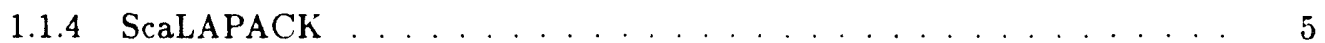

1.2 Target Architectures . . . . . . . . . . . . . . . . . 6

1.3 High-Quality, Reusable, Mathematical Software . . . . . . . . . . . . 7

1.3.1 Performance . . . . . . . . . . . . . . . . . . 7

1.3 .2 Ease-Of-Use . . . . . . . . . . . . . . . . . . . . . 8

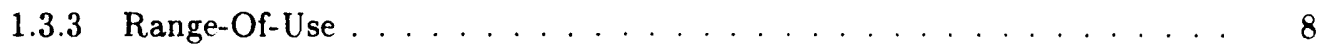

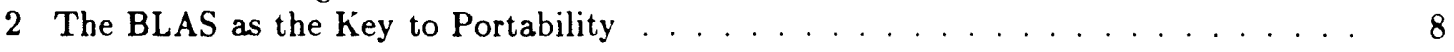

3 Block Algorithms and Their Derivation . . . . . . . . . . . . . . . . . . 10

3.1 Deriving a Block Algorithm . . . . . . . . . . . . . . . . . . 11

3.2 Examples of Block Algorithms in LAPACK . . . . . . . . . . . . . 12

4 LU Factorization . . . . . . . . . . . . . . . . . . . . . 15

4.1 Uses of LU Factorization in Science and Engineering . . . . . . . . . 15

4.2 Derivation of a Block Algorithm for LU Factorization . . . . . . . . . . . 16

5 Data Distribution . . . . . . . . . . . . . . . . . . . . . . . . . . . 18

6 Parallel Implementation . . . . . . . . . . . . . . . . . . . . . . . . 22

7 Optimization, Tuning, and Trade-offs . . . . . . . . . . . . . . . . . . . 30

7.1 Mapping Logical Memory to Physical Memory . . . . . . . . . . . . . . . . . . 30

7.1.1 Assignment of Processes to Processors . . . . . . . . . . . . . . . . . 30

7.1.2 Layout of Local Process Memory . . . . . . . . . . . . . . . . . . . 31

7.2 Tradeoffs between Load Balance and Communication Latency . . . . . . . . . . 32

7.3 Optimality and Pipelining Tradeoffs . . . . . . . . . . . . . . . . . 34

8 Conclusions and Future Research Directions . . . . . . . . . . . . . . . . 36

8.1 Portability, Scalability, and Standards . . . . . . . . . . . . . . 36

8.2 Alternative Approaches . . . . . . . . . . . . . . . . . . . . . . . . . . . . 39

9 References . . . . . . . . . . . . . . . . . . . . 41 


\title{
THE DESIGN OF LINEAR ALGEBRA LIBRARIES FOR HIGH PERFORMANCE COMPUTERS
}

\author{
Jack J. Dongarra \\ David W: Walker
}

\begin{abstract}
This paper discusses the design of linear algebra libraries for high performance computers. Particular emphasis is placed on the devclopment of scalable algorithms for MIMD distributed memory concurrent computers. A brief description of the EISPACK, LINPACK, and LAPACK libraries is given, followed by an outline of ScaLAPACK, which is a distributed memory version of LAPACK currently under development. The importance of block-partitioned algorithms in reducing the frequency of data movement between different levels of hierarchical memory is stressed. The use of such algorithms helps reduce the message startup costs on distributed memory concurrent computers. Other key ideas in our approach are the use of distributed versions of the Level 3 Basic Linear Algebra Subprograms (BLAS) as computational building blocks, and the use of Basic Linear Algebra Communication Subprograms (BLACS) as communication building blocks. Together the distributed BLAS and the BLACS can be used to construct higher-level algorithms, and hide many details of the parallelism from the application developer.

The block-cyclic data distribution is described, and adopted as a good way of distributing block-partitioned matrices. Block-partitioned versions of the Cholesky and LU factorizations are presented, and optimization issues associated with the implementation of the LU factorization algorithm on distributed memory concurrent computers are discussed, together with its performance on the Intel Delta system. Finally, approaches to the design of library interfaces are reviewed.
\end{abstract}




\section{Introduction}

The increasing availability of advanced-architecture computers is having a very significant effect on all spheres of scientific computation, including algorithm resaarch and soft ware development in numerical linear algebra. Linear algebra-in particular, the solution of linear systems of equations-lies at the heart of most calculations in scientific computing. This chapter discusses some of the recent developments in linear algebra designed to exploit these advancedarchitecture computers. Particular attention will be paid to dense factorization routines, such as the Cholesky and LU factorizations, and these will be used as examples to highlight the most important factors that must be considered in designing linear algebra software for advancedarchitecture computers. We use these factorization routines for illustrative purposes not only because they are relatively simple, but also because of their importance in several scientific and engineering applications that make use of boundary element methods. These applications include electromagnetic scattering and computational fluid dynamics problems, as discussed in more detail in Section 4.1.

Much of the work in developing linear algebra software for advanced-architecture computers is motivated by the need to solve large problems on the fastest computers available. In this chapter, we focus on four basic issues: (1) the motivation for the work; (2) the development of standards for use in linear algebra and the building blocks for a library; (3) aspects of algorithm design and parallel implementation; and (4) future directions for research.

For the past 15 years or so, there has been a great deal of activity in the area of algoritlims and software for solving linear algebra problems. The linear algebra community has long recognized the need for help in developing algorithms into software libraries, and several years ago, as a community effort, put together a de facto standard for identifying basic operations required in linear algebra algorithms and software. The hope was that the routines making up this standard, known collectively as the Basic Linear Algebra Subprograms (BLAS), would be efficiently implemented on advanced-architecture computers by many manufacturers, making it possible to reap the portability benefits of having them efficiently implemented on a wide range of machines. This goal has been largely realized.

The key insight of our approach to designing linear algebra algorithms for advanced architecture computers is that the frequency with which data are moved between different levels of the memory hierarchy must be minimized in order to attain high performance. Thus, our main algorithmic approach for exploiting both vectorization and parallelism in our implementations is the use of block-partitioned algorithms, particularly in conjunction with highly-tuned kernels for performing matrix-vector and matrix-matrix operations (the Level 2 and 3 BLAS). In general, the use of block-partitioned algorithms requires data to be moved as blocks, rather than as vectors or scalars, so that although the total amount of data moved is unchanged, the latency 
(or startup cost) associated with the movement is greatly reduced because fewer messages are needed to move the data.

A second key idea is that the performance of an algorithm can be tuned by a user by varying the parameters that specify the data layout. On shared memory machines. this is controlled by the block size. while on distributed memory machines it is controlled by the block size and the configuration of the logical process mesh, as described in more detail in Section 5.

In Section 1, we first give an overview of some of the major software projects aimea at. solving dense linear algebra problems. Next, we describe the types of machine that benefit most from the use of block-partitioned algorithms, and discuss what is meant by high-quality, reusable software for advanced-architecture computers. Section 2 discusses the role of the BLAS in portability and performance on high-performance computers. We discuss the design of these building blocks, and their use in block-partitioned algorithms, in Section 3. Section 4 focuses on the design of a block-partitioned algorithm for LU factorization, and Sections 5, 6, and 7 use this example to illustrate the most important factors in implementing dense linear algebra routines on MIMD, distributed memory, concurrent computers. Section 5 deals with the issue of mapping the data onto the hierarchical memory of a concurrent computer. The iayout of an application's data is crucial in determining the performance and scalability of the parallel code. In Sections 6 and 7 , details of the parallel implementation and optimization issues are discussed. Section 8 presents some future directions for investigation.

\subsection{Dense Linear Algebra Libraries}

Over the past twenty-five years, the first author has been directly involved in the development of several important packages of dense linear algebra software: EISPACK, LINPACK, LAPACK, and the BLAS. In addition, both authors are currently involved in the development of ScaLAPACK, a scalable version of LAPACK for distributed memory concurrent computers. In this section, we give a brief review of these packages-their history, their advantages, and their limitations on high-performance computers.

\subsubsection{EISPACK}

EISPACK is a collection of Fortran subroutines that compute the eigenvalues and eigenvectors of nine classes of matrices: complex general, complex Hermitian, real general, real symmetric, real symmetric banded, real symmetric tridiagonal, special real tridiagonal, generalized real, and generalized real symmetric matrices. In addition, two routines are included that use singular value decomposition to solve certain least-squares problems.

EISPACK is primarily based on a collection of Algol procedures developed in the 1960s and collected by J. H. Wilkinson and C. Reinsch in a volume entitled Linear Algebra in the 
Handbook for Automatac Computaton [5] series. This volume was not designed to cover every possible method of solution: rather, algorithms were chosen on the basis of their generality. elegance, accuracy, speed, or economy of storage.

Since the release of EISPACK in 1972 . over ten thousand copies of the collection have been distributed worldwide.

\subsubsection{LINPACK}

LINPACK is a collection of Fortran subroutines that analyze and solve linear equations and linear least-squares problerns. The package solves linear systems whose matrices are general, banded, symmetric indefinite, symmetric positive definite, triangular, and tridiagonal square. In addition, the package computes the $Q R$ and singular value decompositions of rectangular matrices and applies them to least-squares problems.

LINPACK is organized around four matrix factorizations: LU factorization, pivoted Cholesky factorization, QR factorization, and singular value decomposition. The term LU factorization is used here in a very general sense to mean the factorization of a square matrix into a lower triangular part and an upper triangular part, perhaps with pivoting. These factorizations will be treated at greater length later, when the actual LINPACK subroutines are discussed. But first a digression on organization and factors influencing LINPACK's efficiency is necessary.

LINPACK uses column-oriented algorithms to increase efficiency by preserving locality of reference. This means that if a program references an item in a particular block, the next reference is likely to be in the same block. By column orientation we mean that the LINPACK codes always reference arrays down columns, not across rows. This works because Fortran stores arrays in column major order. Thus, as one proceeds down a column of an array, the memory references proceed sequentially in memory. On the other hand, as one proceeds across a row, the memory references jump across memory, the length of the jump being proportional to the length of a column. The effects of column orientation are quite dramatic: on systems with virtual or cache memories, the LINPACK codes will significantly outperform codes that are not column oriented. We note, however, that textbook examples of matrix algorithms are seldom column oriented.

Another important factor influencing the efficiency of LINPACK is the use of the Level 1 BLAS; there are three effects.

First, the overhead entailed in calling the BLAS reduces the efficiency of the code. This reduction is negligible for large matrices, but it can be quite significant for small matrices. The matrix size at which it becomes unimportant varies from system to system; for square matrices it is typically between $n=25$ and $n=100$. If this seems like an unacceptably large overhead, remember that on many modern systems the solution of a system of order 25 or less is itself a 
negligible calculation. Nonetheless, it cannot be denied that a person whose programs depend critically on solving small matrix problems in inner loops will be better off with BLAS-less versions of the LINPACK codes. Fortunately, the BLAS can be removed from the smaller, more frequently used program in a short editing session.

Second, the BLAS improve the efficiency of programs when they are run on nonoptimizing compilers. This is because doubly subscripted array references in the inner loop of the algorithm are replaced by singly subscripted array references in the appropriate BLAS. The effect can be seen for matri. os of quite small order, and for large orders the savings are quite significant.

Finally, improved efficiency can be achieved by coding a set of BLAS [17] to take advantage of the special features of the computers on which LINPACK is being run. For most computers, this simply means producing machine-language versions. However, the code can also take advantage of more exotic architectural features, such as vector operations.

Further details about the BLAS are presented in Section 2.

\subsubsection{LAPACK}

LAPACK [14] provides routines for solving systems of simultaneous linear equations, leastsquares solutions of linear systems of equations, eigenvalue problems, and singular value problems. The associated matrix factorizations (LU, Cholesky, QR, SVD, Schur, generalized Schur) are also provided, as are related computations such as reordering of the Schur factorizations and estimating condition numbers. Dense and banded matrices are handled, but not general sparse matrices. In all areas, similar functionality is provided for real and complex matrices, in both single and double precision.

The original goal of the LAPACK project was to make the widely used EISPACK and LINPACK libraries run efficiently on shared-memory vector and parallel processors. On these machines, LINPACK and EISPACK are inefficient because their memory access patterns disregard the multilayered memory hierarchies of the machines, thereby spending too much time moving data instead of doing useful floating-point operations. LAPACK addresses this problem by reorganizing the algorithms to use block matrix operations, such as matrix multiplication, in the innermost loops $[3,14]$. These block operations can be optimized for each architecture to account for the memory hierarchy [2], and so provide a transportable way to achieve high efficiency on diverse modern machines. Here we use the term "transportable" instead of "portable" because, for fastest possible performance, LAPACK requires that highly optimized block matrix operations be already implemented on each machine. In other words, the correctness of the code is portable, but high performance is not-if we limit ourselves to a single Fortran source code.

LAPACK can be regarded as a successor to LINPACK and EISPACK. It has virtually all the 
capabilities of these two packages and much more besides. LAPACK improves on LINPACK and EISPACK in four main respects: speed, accuracy, robustness and functionality. While LINPACK and EISPACK are based on the vector operation kernels of the Level 1 BLAS, LAPACK was designed at the outset to exploit the Level 3 BLAS - a set of specifications for Fortran subprograms that do various types of matrix multiplication and the solution of triangular systems with multiple right-hand sides. Because of the coarse granularity of the Level 3 BLAS operations, their use tends to promote high efficiency on many high-performance computers, particularly if specially coded implementations are provided by the manufacturer.

\subsubsection{ScaLAPACK}

The ScaLAPACK software library, scheduled for completion by the end of 1994, will extend the LAPACK library to run scalably on MIMD, distributed memory, concurrent computers $[10,11]$. For such machines the memory hierarchy includes the off-processor memory of other processors, in addition to the hierarchy of registers, cache, and local memory on each processor. Like LAPACK, the ScaLAPACK routines are based on block-partitioned algorithms in order to minimize the frequency of data movement between different levels of the memory hierarchy. The fundamental building blocks of the ScaLAPACK library are distributed memory versions of the Level 2 and Level 3 BLAS, and a set of Basic Linear Algebra Communication Subprograms (BLACS) $[16,26]$ for communication tasks that arise frequently in parallel linear algebra computations. In the ScaLAPACK routines, all interprocessor communication occurs within the distributed BLAS and the BLACS, so the source code of the top software layer of ScaLAPACK looks very similar to that of LAPACK.

We envisage a number of user interfaces to ScaLAPACK. Initially, the interface will be similar to that of LAPACK, with some additional arguments passed to each routine to specify the data layout. Once this is in place, we intend to modify the interface so the arguments to each ScaLAPACK routine are the same as in LAPACK. This will require information about the data distribution of each matrix and vector to be hidden from the user. This may be done by means of a ScaLAPACK initialization routine. This interface will be fully compatible with LAPACK. Provided "dummy" versions of the ScaLAPACK initialization routine and the BLACS are added to LAPACK, there will be no distinction between LAPACK and ScaLAPACK at the application level, though each will link to different versions of the BLAS and BLACS. Following on from this, we will experiment with object-based interfaces for LAPACK and ScaLAPACK, with the goal of developing interfaces compatible with Fortran 90 [10] and $\mathrm{C}++[24]$. 


\subsection{Target Architectures}

The EISPACK and LINPACK software libraries were designed for supercomputers used in the 1970s and early 1980s. such as the CDC-7600. Cyber 205. and (ray-1. These machines featured multiple functional units pipelined for good performance [43]. The CDC-T600 was hasically a high-performance scalar computer. while the Cyber 20i and Cray-1 were early vector computers.

The development of LAPACK in the late 1980s was irtended to make the EISPACK and LINPACK libraries run efficiently on shared memory, vector supercomputers. The ScaLAPACK software library will extend the use of JAPACK to distributed memory concurrent supercomputers. The development of ScaLAPACK began in 1991 and is expected to be completed by the end of 1994.

The underlying concept of both the LAPACK and ScaLAPACK libraries is the use of block-partitioned algorithms to minimize data movement between different levels in hierarchical memory. Thus, the ideas discussed in this chapter for developing a library for dense linear algebra computations are applicable to any computer with a hierarchical memory that (1) imposes a sufficiently large startup cost on the movement of data between different levels in the hierarchy, and for which (2) the cost of a context switch is too great to make fine grain size multithreading worthwhile. Our target machines are, therefore, medium and large grain size advanced-architecture computers. These include "traditional" shared memory, vector supercomputers, such as the Cray Y-MP and C90, and MIMD distributed memory concurrent supercomputers, such as the Intel Paragon, and Thinking Machines' CM-5, and the more recently announced IBM SP1 and Cray T3D concurrent systems. Since these machines have only very recently become available, most of the ongoing development of the ScaLAPACK library is being done on a 128-node Intel iPSC/860 hypercube and on the 520-node Intel Delta system.

The Intel Paragon supercomputer can have up to 2000 nodes, each consisting of an i860 processor and a communications processor. The nodes each have at least $16 \mathrm{Mby}$ ues of memory, and are connected by a high-speed network with the topology of a two-dimensional mesh. The CM-5 from Thinking Machines Corporation [53] supports both SIMD and MIMD programming models, and may have up to $16 \mathrm{k}$ processors, though the largest CM-5 currently installed has 1024 processors. Each CM-5 node is a Sparc processor and up to 4 associated vector processors. Point-to-point communication between nodes is supported by a data network with the topology of a "fat tree" [46]. Global communication operations, such as synchronization and reduction, are supported by a separate control network. The IBM SP1 system is based on the same RISC chip used in the IBM RS/6000 workstations and uses a multistage switch to connect processors. The Cray T3D uses the Alpha chip from Digital Equipment Corporation, and connects the processors in a three-dimensional torus. 
Future advances in compiler and hardware technologies in the mid to late 1990) are expected to make multithreading a viable approach for masking communication costs. Since the blocks in a block-partitioned algorithm can be regarded as separate threads. our approach will still be applicable on machines that exploit medium and coarse grain size multithreading.

\subsection{High-Quality, Reusable, Mathematical Software}

In developing a library of high-quality subroutines for dense linear algebra computations the design goals fall into three broad classes:

- performance

- ease-of-use

- range-of-use

\subsubsection{Performance}

Two important performance metrics are concurrent efficiency and scalability. We seek good performance characteristics in our algorithms by eliminating, as much as possible, overhead due to load imbalance, data movement, and algorithm restructuring. The way the data are distributed (or decomposed) over the memory hierarchy of a computer is of fundamental importance to these factors. Concurrent efficiency, $\epsilon$, is defined as the concurrent speedup per processor [32], where the concurrent speedup is the execution time, $T_{\text {seq }}$, for the best sequential algorithm running on one processor of the concurrent computer, divided by the execution time, $T$, of the parallel algorithm running on $N_{p}$ processors. When direct methods are used, as in LU factorization, the concurrent efficiency depends on the problem size and the number of processors, so on a given parallel computer and for a fixed number of processors, the running time should not vary greatly for problems of the same size. Thus, we may write,

$$
\epsilon\left(N, N_{p}\right)=\frac{1}{N_{p}} \frac{T_{\mathrm{seq}}(N)}{T\left(N^{\prime}, N_{p}\right)}
$$

where $N$ represcats the problem size. In dense linear algebra computations, the execution time is usually dominated by the floating-point operation count, so the concurrent efficiency is related to the performance, $G$, measured in floating-point operations per second by,

$$
G\left(N, N_{p}\right)=\frac{N_{p}}{t_{\text {calc }}} \epsilon\left(N, N_{p}\right)
$$

where $t_{\text {calc }}$ is the time for one floating-point operation. For iterative routines, such as eigensolvers, the number of iterations, and hence the execution time, depends not only on the problem size, but also on other characteristics of the input data, such as condition number. A parallel 
algorithm is said to be scalable [3i] if the concurrent eficiency depends on the problem size and number of processors only through their ratio. This ratio is simply the problem size per processor. often referred to as the granularity. Thus, for a scalable algorithm. the concurrent efficiency is constant as the number of processors increases while keeping the granularity fixed. Alternatively, Eq. 2 shows that this is equivalent to saying that, for a scalable algorithm, the performance depends linearly on the number of processors for fixed granularity.

\subsubsection{Ease-Of-Use}

Ease-of-use is concerned with factors such as portability and the user interface to the library: Portability, in its most inclusive sense, means that the code is written in a standard language, such as Fortran, and that the source code can be compiled on an arbitrary machine to produce a program that will run correctly. We call this the "mail-order software" model of portability, since it reflects the model used by software servers such as netlib [20]. This notion of portability is quite demanding. It requires that all relevant properties of the computer's arithmetic and architecture be discovered at runtime within the confines of a Fortran code. For example, if it is important to know the overflow threshold for scaling purposes, it must be determined at runtime without overflowing, since overflow is generally fatal. Such demands have resulted in quite large and sophisticated programs $[28,44]$ which must be modified frequently to deal with new architectures and software releases. This "mail-order" notion of software portability also means that codes generally must be written for the worst possible machine expected to be used, thereby often degrading performance on all others. Ease-of-use is also enhanced if implementation details are largely hidden from the user, for example, through the use of an object-based interface to the library [24].

\subsubsection{Range-Of-Use}

Range-of-use may be gauged by how numerically stable the algorithms are over a range of input problems, and the range of data structures the library will support. For example, LINPACK and EISPACK deal with dense matrices stored in a rectangular array, packed matrices where only the upper or lower half of a symmetric matrix is stored, and banded matrices where only the nonzero bands are stored. In addition, some special formats such as Householder vectors are used internally to represent orthogonal matrices. There are also sparse matrices, which may be stored in many different ways; but in this paper we focus on dense and banded matrices, the mathematical types addressed by LINPACK, EISPACK, and LAPACK.

\section{The BLAS as the Key to Portability}

At least three factors affect the performance of portable Fortran code. 
1. Vectorizaticii. Designing vectorizable algorithms in linear algebra is usually straightforward. Indeed. for many computations there are several variants. all vectorizable but with different characteristics in performance (see. for example. [15]). Linear algebra algorith ns can approach the peak performance of many machines-principally because peak performance depends on some form of chaining of vector addition and multiplication operations, and this is just what the algorithms require. However, when the algorithms are realized in straightforward Fortran 77 code, the performance may fall well short of the expected level, usually because vectorizing Fortran compilers fail to minimize the number oi memory reference; - that is, the number of vector load and store operations.

2. Data movenuent. What often limits the actual performance of a vector, or scalar, floating-point unit is the rate of transfer of data between different levels of memory in the machine. Examples include the transfer of vector operands in and out of vector registers, the transfer of scalar operands in and out of a high-speed scalar processor, the movement of data between main memory and a high-speed cache or local memory, paging between actual memory and disk storage in a virtual memory system, and interprocessor comn unicaticn on a distributed memory concurrent computer.

3. Parallelism. The nested loop structure of most linear algebra algorithms offers considerable scope for loop-based parallelism. This is the principal type of parallelism that LAPACK and ScaLAPACK presently aim to exploit. On shared memory concurrent computers, this type of parallelism can sometimes be generated automatically by a compiler, but often requires the insertion of compiler directives. On distributed memory concurrent computers, data must be moved between processors. This is usually done by explicit calls to message passing routines, although parallel language extensions such as Coherent Parallel C [31] and Split-C [13] do the message passing implicitly.

The question arises, "How can we achieve sufficient control over these three factors to obtain the levels of performance that machines can offer?" The answer is through use of the BLAS.

There are now three levels of BLAS:

Level 1 BLAS [15]: for vector operations, such as $y-\alpha x+y$

Level 2 BLAS [18]: for matrix-vector operations, such as $y-\alpha A x+\beta y$

Level 3 BLAS [17]: for matrix-matrix operations, such as $C-\alpha A B+\beta C$.

Here, $A, B$ and $C$ are matrices, $x$ and $y$ are vectors, and $\alpha$ and $\beta$ are scalars

The Level 1 BLAS are used in LAPACK, but for convenience rather than for performance: they perform an insignificant fraction of the computation, and they cannot achieve high efficiency on most modern supercomputers. 
Table 1: Speed (Megaflops) of Level 2 and Level 3 BLAS Operations on a (RAY Y-MI'. All matrices are of order $500: l^{\circ}$ is upper triangular.

\begin{tabular}{|l|r|r|r|r|}
\hline Tumber of processors: & 1 & 2 & 4 & 8 \\
\hline \hline Level 2: $y-a A x+3 y$ & 311 & 611 & 1197 & 2285 \\
\hline Level 3: $C-a A B+3 C$ & 312 & 623 & 1247 & 2425 \\
\hline \hline Level 2: $x-C$ & 293 & 544 & 898 & 1613 \\
\hline Level 3: $B-C B$ & 310 & 620 & 1240 & 2425 \\
\hline \hline Level 2: $x-!^{-1} x$ & 272 & 374 & 479 & 584 \\
\hline Level 3: $B-U^{-1} B$ & 309 & 618 & 1235 & 2398 \\
\hline
\end{tabular}

The Level 2 BLAS can achieve near-peak performance on many vector processors, such as a single processor of a CRAY X-MP or Y-MP, or Convex C-2 machine. However, on other vector processors such as a CRAY-2 or an IBM $3090 \mathrm{VF}$, the performance of the Level 2 BLAS is limited by the rate of data movement between different levels of memory.

The Level 3 BLAS overcome this limitation. This third level of BLAS performs $O\left(n^{3}\right)$ floating-point operations on $O\left(n^{2}\right)$ data, whereas the Level 2 BLAS perform only $O\left(n^{2}\right)$ operations on $O\left(n^{2}\right)$ data. The Level 3 BLAS also allow us to exploit parallelism in a way that is transparent to the software that calls them. While the Level 2 BLAS offer some scope for exploiting parallelism, greater scope is provided by the Level 3 BLAS, as Table 1 illustrates.

\section{Block Algorithms and Their Derivation}

It is comparatively straightforward to recode many of the algorithms in LINPACK and EISPACK so that they call Level 2 BLAS. Indeed, in the simplest cases the same floating-point operations are done, possibly even in the same order it is just a matter of reorganizing the software. To illustrate this point, we consider the Cholesky factorization algorithm used in the LINPACK routine SPOFA, which factorizes a symmetric positive definite matrix as $A=U^{T} U$. We consider Cholesky factorization because the algorithm is simple, and no pivoting is required. In Section 4 we shall consider the slightly more complicated example of LU factorization.

Suppose that after $j-1$ steps the block $A_{00}$ in the upper lefthand corner of $A$ has been factored as $A_{00}=U_{00}^{T} U_{00}$. The next row and column of the factorization can then be computed by writing $A=U^{T} U$ as

$$
\left(\begin{array}{ccc}
A_{00} & b_{j} & A_{02} \\
\cdot & a_{j j} & c_{j}^{T} \\
\cdot & \cdot & A_{22}
\end{array}\right)=\left(\begin{array}{ccc}
U_{011}^{T} & 0 & 0 \\
v_{j}^{T} & u_{j j} & 0 \\
U_{02}^{T} & u_{j} & U_{22}^{T}
\end{array}\right)\left(\begin{array}{ccc}
U_{00} & v_{j} & U_{02} \\
0 & u_{j j} & u_{j}^{T} \\
0 & 0 & U_{22}
\end{array}\right)
$$

where $b_{j}, c_{j}, v_{j}$, and $w_{j}$ are column vectors of length $j-1$, and $a_{j}$ and $u_{j j}$ are scalars. Equating 
coefficients of the $j^{\text {th }}$ column. we obtain

$$
\begin{aligned}
b_{j} & =l_{00}^{T} v_{j} \\
a_{j j} & =v_{j}^{T} v_{j}+u_{j j}^{2} .
\end{aligned}
$$

Since $U_{00}$ has already been computed, we can compute $v_{j}$ and $u_{j j}$ from the equations

$$
\begin{aligned}
U_{00}^{T} v_{j} & =b_{j} \\
u_{j j}^{2} & =a_{j j}-v_{j}^{T} v_{j} .
\end{aligned}
$$

The body of the code of the LINPACK routine SPOFA that implements the above method is shown in Figure 1. The same computation recoded in "LAPACK-style" to use the Level 2 BLAS routine STRSV (which solves a triangular system of equations) is shown in Figure 2. The call to STRSV has replaced the loop over $\mathrm{K}$ which made several calls to the Level 1 BLAS routine SDOT. (For reasons given below, this is not the actual code used in LAPACK - hence the term "LAPACK-style".)

This change by itself is sufficient to result in large gains in performance on a number of machines-for example, from 72 to 251 megaflops for a matrix of order 500 on one processor of a CRAY Y-MP. Since this is $81 \%$ of the peak speed of matrix-matrix multiplication on this processor, we cannot hope to do very much better by using Level 3 BLAS.

We can, however, restructure the algorithm at a deeper level to exploit the faster speed of the Level 3 BLAS. This restructuring involves recasting the algorithm as a block algorithm-that is, an aigorithm that operates on blocks or submatrices of the original matrix.

\subsection{Deriving a Block Algorithm}

To derive a block form of Cholesky factorization, we partition the matrices as shown in Figure 4 , in which the diagonal blocks of $A$ and $U$ are square, but of differing sizes. We assume that the first block has already been factored as $A_{00}=U_{00}^{T} U_{00}$, and that we now want to determine the second block column of $U$ consisting of the blocks $U_{01}$ and $U_{11}$. Equating submatrices in the second block of columns, we obtain

$$
\begin{aligned}
& A_{01}=U_{00}^{T} U_{01} \\
& A_{11}=U_{01}^{T} U_{01}+U_{11}^{T} U_{11} .
\end{aligned}
$$

Hence, since $U_{00}$ has already been computed, we can compute $U_{01}$ as the solution to the equation

$$
U_{00}^{T} U_{01}=A_{01}
$$


Table 2: Speed (Megaflops) of Cholesky Factorization $A=I^{*} l^{\cdot}$ for $n=500$

\begin{tabular}{|l|r|r|r|}
\hline & $\begin{array}{r}\text { IBM 3090 VF, } \\
1 \text { proc. }\end{array}$ & $\begin{array}{r}\text { CRAY Y-MP, } \\
1 \text { proc. }\end{array}$ & $\begin{array}{r}\text { CRAY Y-MP. } \\
8 \text { proc. }\end{array}$ \\
\hline$j$-variant: LINPACK & 23 & 72 & 72 \\
$j$-variant: using Level 2 BLAS & 24 & 251 & 378 \\
$j$-variant: using Level 3 BLAS & 49 & 287 & 1225 \\
$i$-variant: using Level 3 BLAS & 50 & 290 & 1414 \\
\hline
\end{tabular}

by a call to the Level 3 BLAS routine STRSM; and then we can compute $U_{11}$ from

$$
U_{11}^{T} U_{11}=A_{11}-U_{01}^{T} U_{01}
$$

This involves first updating the symmetric submatrix $A_{11}$ by a call to the Level 3 BLAS routine SSYRK, and then computing its Cholesky factorization. Since Fortran does not allow recursion, a separate routine must be called (using Level 2 BLAS rather than Level 3), named SPOTF2 in Figure 3. In this way, successive blocks of columns of $U$ are cornputed. The LAPACK-style code for the block algorithm is shown in Figure 3. This code runs at 49 megaflops on an IBM 3090, more than double the speed of the LINPACK code. On a CRAY Y-MP, the use of Level 3 BLAS squeezes a little more performance out of one processor, but makes a large improvement when using all 8 processors.

But that is not the end of the story, and the code given above is not the code actually used in the LAPACK routine SPOTRF. We mentioned earlier that for many linear algebra computations there are several algorithmic variants, often referred to as $i-, j-$, and $k$-variants, according to a convention introduced in [15] and used in [36]. The same is true of the corresponding block algorithms.

It turns out that the $j$-variant chosen for LINPACK, and used in the above examples, is not the fastest on many machines, because it performs most of the work in solving triangular systems of equations, which can be significantly slower than matrix-matrix multiplication. The variant actually used in LAPACK is the $i$-variant, which relies on matrix-matrix multiplication for most of the work.

Table 2 summarizes the results.

\subsection{Examples of Block Algorithms in LAPACK}

Having discussed in detail the derivation of one particular block algorithm, we now describe examples of the performance achieved with two well-known block algorithms: LU and Cholesky factorizations. No extra floating-point operations nor extra working storage are required for either of these simple block algorithms. (See Gallivan et al. [33] and Dongarra et al. [19] for 


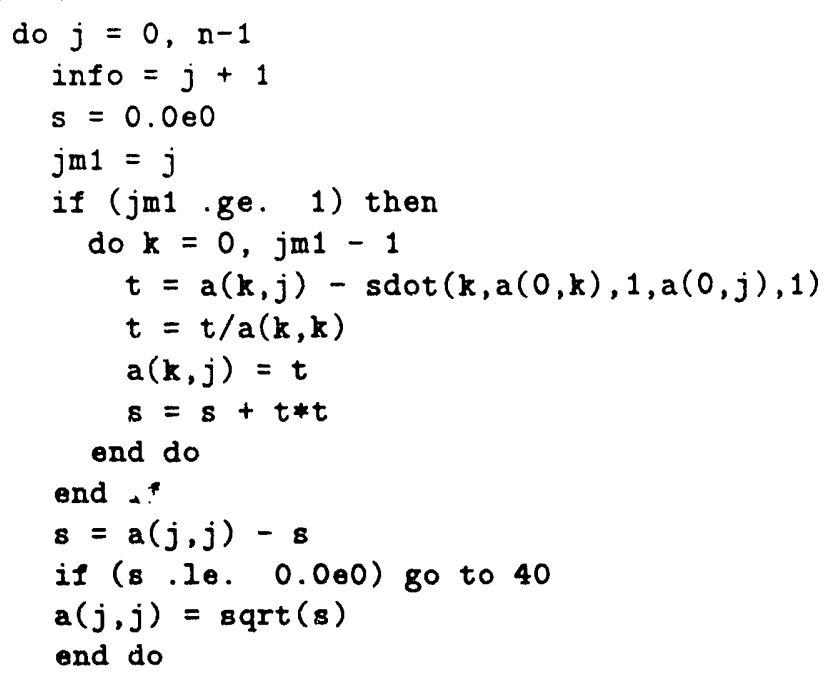

Figure 1: The body of the LINPACK routine SPOFA for Cholesky factorization.

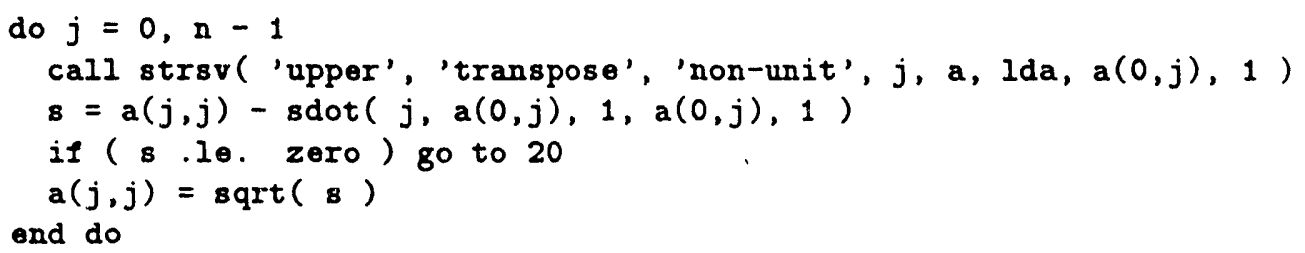

Figure 2: The body of the "LAPACK-style" routine SPOFA for Cholesky factorization.

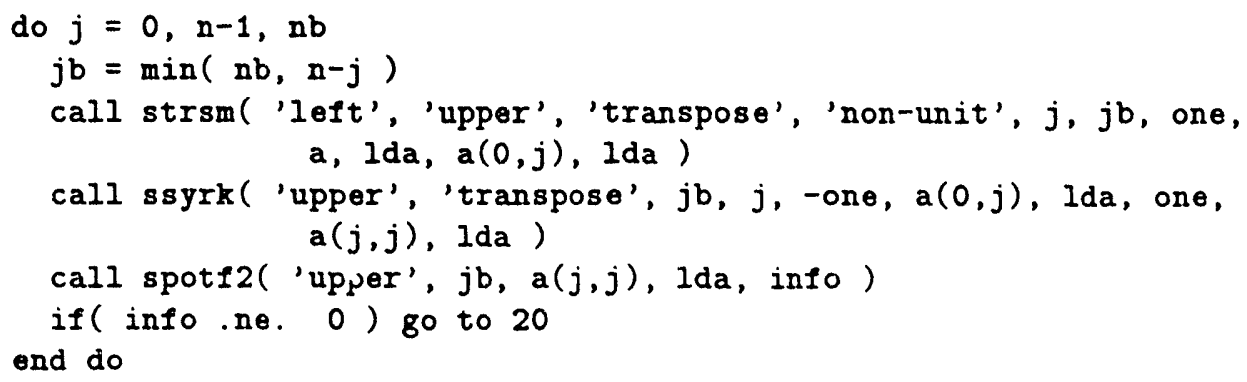

Figure 3: The body of the "LAPACK-style" routine SPOFA for block Cholesky factorization. In this code fragment, nb denotes the width of the blocks. 


\begin{tabular}{|l|l|l|}
\hline$A_{00}$ & $A_{01}$ & $A_{02}$ \\
\hline$A_{01}^{\top}$ & $A_{11}$ & $A_{12}$ \\
\hline$A_{02}^{\top}$ & $A_{12}^{\top}$ & $A_{22}$
\end{tabular}$=$\begin{tabular}{|l|l|l|l|l|l|}
\hline$U_{00}^{\top}$ & 0 & 0 \\
\hline$U_{01}^{\top}$ & $U_{11}^{\top}$ & 0 & $U_{00}$ & $U_{01}$ & $U_{02}$ \\
\hline 0 & $U_{02}^{\top}$ & $U_{12}^{\top}$ & $U_{22}^{\top}$
\end{tabular}$*$\begin{tabular}{|c|c|c|}
\hline 0 & 0 & $U_{12}$ \\
\hline
\end{tabular}

Figure 4: Partitioning of $A, U^{T}$, and $U$ into blocks. It is assumed that the first block has already been factored as $A_{00}=U_{00}^{T} U_{00}$, and we next want to determine the block column consisting of $U_{01}$ and $U_{11}$. Noie that the diagonal blocks of $A$ and $U$ are square matrices.

Table 3: Speed (Megaflops) of SGETRF/DGETRF for Square Matrices of Order $n$

\begin{tabular}{|l|c|r||r|r|r|r|r|}
\hline Machine & \multicolumn{1}{c|}{$\begin{array}{c}\text { No. of } \\
\text { processors }\end{array}$} & \multicolumn{1}{c|}{\begin{tabular}{c} 
slock \\
\cline { 4 - 7 }
\end{tabular}} & \multicolumn{1}{|c|}{ size } & \multicolumn{5}{|c|}{ Values of $n$} \\
\hline IBM RISC/6000-530 & 1 & 32 & 19 & 200 & 300 & 400 & 500 \\
Alliant FX/8 & 8 & 16 & 9 & 26 & 32 & 31 & 33 \\
IBM 3090J VF & 1 & 64 & 23 & 41 & 52 & 58 & 57 \\
Convex C-240 & 4 & 64 & 31 & 60 & 82 & 100 & 112 \\
CRAY Y-MP & 1 & 1 & 132 & 219 & 254 & 272 & 283 \\
CRAY-2 & 1 & 64 & 110 & 211 & 292 & 318 & 358 \\
Siemens/Fujitsu VP 400-EX & 1 & 64 & 46 & 132 & 222 & 309 & 397 \\
NEC SX2 & 1 & 1 & 118 & 274 & 412 & 504 & 577 \\
CRAY Y-MP & 8 & 64 & 195 & 556 & 920 & 1188 & 1408 \\
\hline
\end{tabular}

surveys of algorithms for dense linear algebra on high-performance computers.)

Table 3 illustrates the speed of the LAPACK routine for LU factorization of a real matrix, SGETRF in single precision on CRAY machines, and DGETRF in double precision on all other machines. Thus, 64-bit floating-point arithmetic is used on all machines tested. A block size of 1 means that the unblocked algorithm is used, since it is faster than - or at least as fast as - a block algorithm.

LAPACK is designed to give high efficiency on vector processors, high-performance "superscalar" workstations, and shared memory multiprocessors. LAPACK in its present form is less likely to give good performance on other types of parallel architectures (for example, massively parallel SIMD machines, or MIMD distributed memory machines), but the ScaLAPACK project, described in Section 1.1.4, is intended to adapt LAPACK to these new architectures. LAPACK can also be used satisfactorily on all types of scalar machines (PCs, workstations, mainframes).

Table 4 gives similar results for Cholesky factorization, extending the results given in Table 2. 
Table 4: Speed (Megaflops) of SPOTRF/DPOTRF for Matrices of Order $n$. Here LPLO $=$ ' $\mathrm{U}$ '. so the factorization is of the form $A=l^{r} L$ '.

\begin{tabular}{|c|c|c|c|c|c|c|c|}
\hline \multirow[t]{2}{*}{ Machine } & \multirow{2}{*}{$\begin{array}{c}\text { No. of } \\
\text { processors }\end{array}$} & \multirow{2}{*}{$\begin{array}{l}\text { Block } \\
\text { size }\end{array}$} & \multicolumn{5}{|c|}{ Values of $n$} \\
\hline & & & 100 & 200 & 300 & 400 & 500 \\
\hline IBM RISC $/ 6000-530$ & 1 & 32 & 21 & 29 & 34 & 36 & 38 \\
\hline Alliant $\mathrm{FX} / 8$ & 8 & 16 & 10 & 27 & 40 & 49 & 52 \\
\hline IBM $3090 \mathrm{~J}$ VF & 1 & 48 & 26 & 43 & 56 & 62 & 67 \\
\hline Convex C-240 & 4 & 64 & 32 & 63 & 82 & 96 & 103 \\
\hline CRAY Y-MP & 1 & 1 & 126 & 219 & 257 & 275 & 285 \\
\hline CRAY-2 & 1 & 64 & 109 & 213 & 294 & 318 & 362 \\
\hline Siemens/Fujitsu VP 400-EX & 1 & 1 & 53 & 145 & 237 & 312 & 369 \\
\hline NEC SX2 & 1 & 1 & 155 & 387 & 589 & 719 & 819 \\
\hline CRAY Y-MP & 8 & 32 & 146 & 479 & 845 & 1164 & 1393 \\
\hline
\end{tabular}

LAPACK, like LINPACK, provides LU and Cholesky factorizations of band matrices. The LINPACK algorithms can easily be restructured to use Level 2 BLAS, though restructuring has little effect on performance for matrices of very narrow bandwidth. It is also possible to use Level 3 BLAS, at the price of doing some extra work with zero elements outside the band [22]. This process becomes worthwhile for large matrices and semi-bandwidth greater than 100 or so.

\section{LU Factorization}

In this section, we first discuss the uses of dense $L U$ factorization in several fields. We next develop a block-partitioned version of the $k$, or right-looking, variant of the $L U$ factorization algorithm. In subsequent sections, the parallelization of this algorithm is described in detail in order to highlight the issues and considerations that must be taken into account in developing an efficient, scalable, and transportable dense linear algebra library for MIMD, distributed memory, concurrent computers.

\subsection{Uses of LU Factorization in Science and Engineering}

A major source of large dense linear systems is problems involving the solution of boundary integral equations. These are integral equations defined on the boundary of a region of interest. All examples of practical interest compute some intermediate quantity on a two-dimensional boundary and then use this information to compute the final desired quantity in three-dimensional space. The price one pays for replacing three dimensions with two is that what started as a sparse problem in $O\left(n^{3}\right)$ variables is replaced by a dense problem in $O\left(n^{2}\right)$.

Dense systems of linear equations are found in numerous applications, including: 
- airplane wing design;

- radar cross-section studies;

- flow around ships and other off-shore constructions:

- diffusion of solid bodies in a liquid:

- noise reduction; and

- diffusion of light through small particles.

The electromagnetics community is a major user of dense linear systems solvers. Of particular interest to this community is the solution of the so-called radar cross-section problem. In this problem, a signal of fixed frequency bounces off an object; the goal is to determine the intensity of the reflected signal in all possible directions. The underlying differential equation may vary, depending on the specific problem. In the design of stealth aircraft, the principal equation is the Helmholtz equation. To solve this equation, researchers use the method of moments $[38,56]$. In the case of fluid flow, the problem often involves solving the Laplace or Poisson equation. Here, the boundary integral solution is known as the panel method [40,41], so named from the quadrilaterals that discretize and approximate a structure such as an airplane. Generally, these methods are called boundary element methods.

Use of these methods produces a dense linear system of size $O(N)$ by $O(N)$, where $N$ is the number of boundary points (or panels) being used. It is not unusual to see size $3 N$ by $3 N$, because of three physical quantities of interest at every boundary element.

A typical approach to solving such systems is to use LU factorization. Each entry of the matrix is computed as an interaction of two boundary elements. Often, many integrals must be computed. In many instances, the time required to compute the matrix is considerably larger than the time for solution.

Only the builders of stealth technology who are interested in radar cross-sections are considering using direct Gaussian elimination methods for solving dense linear systems. These systems are always symmetric and complex, but not Hermitian.

For further information on various methods for solving large dense linear algebra problems that arise in computational fluid dynamics, see the report by Alan Edelman [30].

\subsection{Derivation of a Block Algorithm for LU Factorization}

Suppose the $M \times N$ matrix $A$ is partitioned as shown in Figure 5, and we seek a factorization $A=L U$, where the partitioning of $L$ and $U$ is also shown in Figure 5 . Then we may write,

$$
L_{00} U_{00}=A_{00}
$$




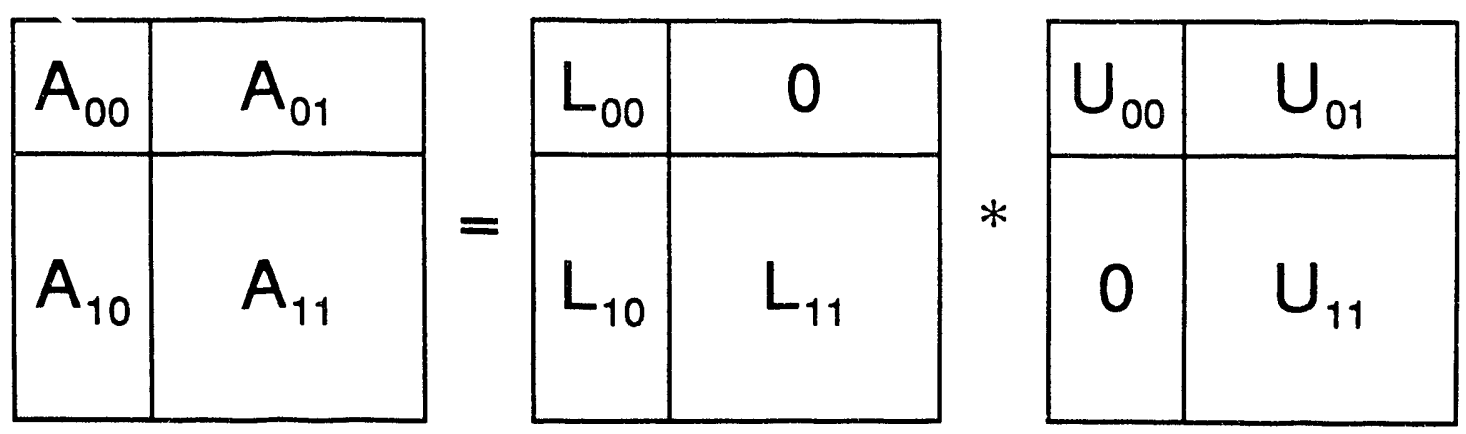

Figure 5: Block LU factorization of the partitioned matrix $A$. $A_{00}$ is $r \times r, A_{01}$ is $r \times(N-r)$, $A_{10}$ is $(M-r) \times r$, and $A_{11}$ is $(M-r) \times(N-r) . L_{00}$ and $L_{11}$ are lower triangular matrices with 1 's on the main diagonal, and $U_{00}$ and $U_{11}$ are upper triangular matrices.

$$
\begin{aligned}
L_{10} U_{00} & =A_{10} \\
L_{00} U_{01} & =A_{01} \\
L_{10} U_{01}+L_{11} U_{11} & =A_{11}
\end{aligned}
$$

where $A_{00}$ is $r \times r, A_{01}$ is $r \times(N-r), A_{10}$ is $(M-r) \times r$, and $A_{11}$ is $(M-r) \times(N-r) . L_{00}$ and $L_{11}$ are lower triangular matrices with 1 s on the main diagonal, and $U_{00}$ and $U_{11}$ are upper triangular matrices.

Equations 3 and 4 taken together perform an LU factorization on the first $M \times r$ panel of $A$ (i.e., $A_{00}$ and $A_{10}$ ). Once this is completed, the matrices $L_{00}, L_{10}$, and $U_{00}$ are known, and the lower triangular system in Eq. 5 can be solved to give $U_{01}$. Finally, we rearrange Eq. 6 as,

$$
A_{11}^{\prime}=A_{11}-L_{10} U_{01}=L_{11} U_{11}
$$

From this equation we see that the problem of finding $L_{11}$ and $U_{11}$ reduces to finding the $L U$ factorization of the $(M-r) \times(N-r)$ matrix $A_{11}^{\prime}$. This can be done by applying the steps outlined above to $A_{11}^{\prime}$ instead of to $A$. Repeating these steps $K$ times, where

$$
K=\min (\lceil M / r\rceil,\lceil N / r\rceil)
$$

we obtain the LU factorization of the original $M \times N$ matrix $A$. For an in-place algorithm, $A$ is overwritten by $L$ and $U$ - the $1 \mathrm{~s}$ on the diagonal of $L$ do not need to be stored explicitly. Similarly, when $A$ is updated by Eq. 7 this may also be done in place.

After $k$ of these $K$ steps, the first $k r$ columns of $L$ and the first $k r$ rows of $U$ have been evaluated, and matrix $A$ has been updated to the form shown in Figure 6, in which panel $B$ is $(M-k r) \times r$ and $C$ is $r \times(N-(k-1) r)$. Step $k+1$ then proceeds as follows, 


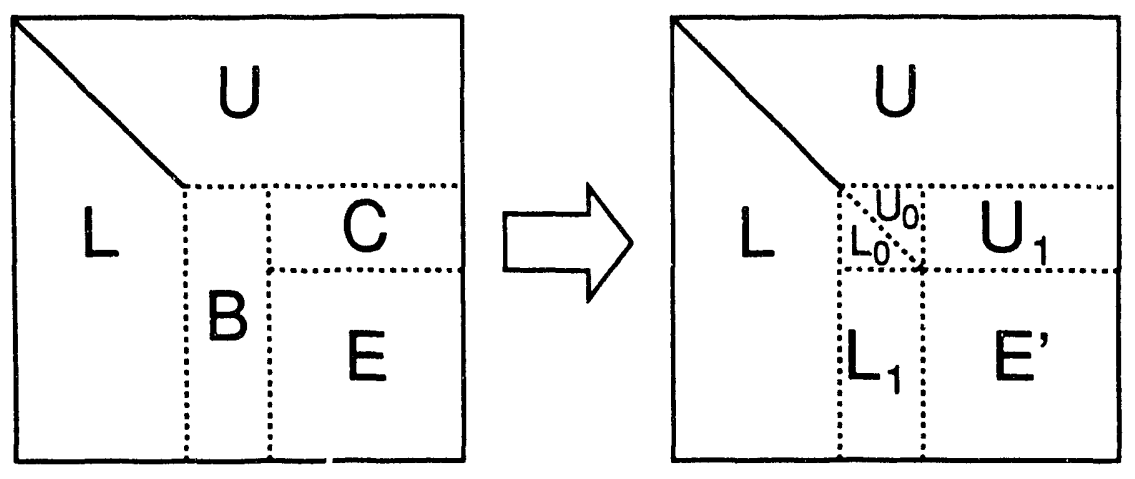

Figure 6: Stage $k+1$ of the block LU factorization algorithm showing how the panels $B$ and $C$, and the trailing submatrix $E$ are updated. The trapezoidal submatrices $L$ and $U$ have already been factored in previous steps. $L$ has $k r$ columns, and $U$ has $k r$ rows. In the step shown another $r$ columns of $L$ and $r$ rows of $U$ are evaluated.

1. factor $B$ to form the next panel of $L$, performing partial pivoting over rows if necessary (see Figure 14). This evaluates the matrices $L_{0}, L_{1}$, and $U_{0}$ in Figure 6.

2. solve the triangular system $L_{0} U_{1}=C$ to get the next row of blocks of $U$.

3. do a rank- $r$ update on the trailing submatrix $E$, replacing it with $E^{\prime}=E-L_{1} U_{1}$.

The LAPACK implementation of this form of LU factorization uses the Level 3 BLAS routines XTRSM and XGEMM to perform the triangular solve and rank- $r$ update. We can regard the algorithm as acting on matrices that have been partitioned into blocks of $r \times r$ elements, as shown in Figure 7.

\section{Data Distribution}

The fundamental data object in the LU factorization algorithm presented in Section 4.2 is a block-partitioned matrix. In this section, we describe the block-cyclic method for distributing such a matrix over a two-dimensional mesh of processes, or template. In general, each process has an independent thread of control, and with each process is associated some local memory directly accessible only by that process. The assignment of these processes to physical processors is a machine-dependent optimization issue, and will be considered later in Section 7 .

An important property of the class of data distribution we shall use is that independent decompositions are applied over rows and columns. We shall, therefore, begin by considering the distribution of a vector of $M$ data objects over $P$ processes. This can be described by a mapping of the global index, $m$, of a data object to an index pair $(p, i)$, where $p$ specifies the 


\begin{tabular}{|l|l|l|l|l|l|}
\hline$A_{0,0}$ & $A_{0,1}$ & $A_{0,2}$ & $A_{0,3}$ & $A_{0,4}$ & $A_{0,5}$ \\
\hline$A_{1,0}$ & $A_{1,1}$ & $A_{1,2}$ & $A_{1,3}$ & $A_{1,4}$ & $A_{1,5}$ \\
\hline$A_{2,0}$ & $A_{2,1}$ & $A_{2,2}$ & $A_{2,3}$ & $A_{2,4}$ & $A_{2,5}$ \\
\hline$A_{3,0}$ & $A_{3,1}$ & $A_{3,2}$ & $A_{3,3}$ & $A_{3,4}$ & $A_{3,5}$ \\
\hline$A_{4,0}$ & $A_{4,1}$ & $A_{4,2}$ & $A_{4,3}$ & $A_{4,4}$ & $A_{4,5}$ \\
\hline$A_{5,0}$ & $A_{5,1}$ & $A_{5,2}$ & $A_{5,3}$ & $A_{5,4}$ & $A_{5,5}$ \\
\hline
\end{tabular}

Figure 7: Block-partitioned matrix $A$. Each block $A_{i, j}$ consists of $r \times r$ matrix elements.

process to which the data object is assigned, and $i$ specifies the location in the local memory of $p$ at which it is stored. We shall assume $0 \leq m<M$ and $0 \leq p<P$.

Two common decompositions are the block and the cyclic decompositions $[55,32]$. The block decomposition, that is often used when the computational load is distributed homogeneously over a regular data structure such as a Cartesian grid, assigns contiguous entries in the global vector to the processes in blocks.

$$
m \mapsto(\lfloor m / L\rfloor, m \bmod L)
$$

where $L=\lceil M / P\rceil$. The cyclic decomposition (also known as the wrapped or scattered decomposition) is commonly used to improve load balance when the computational load is distributed inhomogeneously over a regular data structure. The cyclic decomposition assigns consecutive entries in the global vertor to successive different processes,

$$
m \mapsto(m \bmod P,\lfloor m / P\rfloor)
$$

Examples of the block and cyclic decompositions are shown in Figure 8.

The block cyclic decomposition is a generalization of the block and cyclic decompnsitions in which blocks of consecutive data objects are distributed cyclically over the processes. In the block cyclic decomposition the mapping of the global index, $m$, can be expressed as $m \mapsto(p, b, i)$, where $p$ is the process number, $b$ is the block number in process $p$, and $i$ is the index within block $b$ to which $m$ is mapped. Thus, if the number of data objects in a block is $r$, the block 


\begin{tabular}{|lllllllllll|}
\hline $\mathrm{m}$ & 0 & 1 & 2 & 3 & 4 & 5 & 6 & $\mathrm{i}$ & 8 & 9 \\
\hline $\mathrm{p}$ & 0 & 0 & 0 & 0 & 1 & 1 & 1 & 1 & 2 & 2 \\
\hline $\mathrm{i}$ & 0 & 1 & 2 & 3 & 0 & 1 & 2 & 3 & 0 & 1 \\
\hline
\end{tabular}

(a) Block

\begin{tabular}{|c|cccccccccc|}
\hline $\mathrm{m}$ & 0 & 1 & 2 & 3 & 4 & 5 & 6 & 7 & 8 & 9 \\
\hline $\mathrm{p}$ & 0 & 1 & 2 & 0 & 1 & 2 & 0 & 1 & 2 & 0 \\
\hline $\mathrm{i}$ & 0 & 0 & 0 & 1 & 1 & 1 & 2 & 2 & 2 & 3 \\
\hline
\end{tabular}

(b) Cyclic

Figure 8: Examples of block and cyclic decompositions of $M=10$ data objects over $P=3$ processes.

\begin{tabular}{|c|cccccccccccccccccccccccc|}
\hline $\mathrm{m}$ & 0 & 1 & 2 & 3 & 4 & 5 & 6 & 7 & 8 & 9 & 10 & 11 & 12 & 13 & 14 & 15 & 16 & 17 & 18 & 19 & 20 & 21 & 22 \\
\hline $\mathrm{p}$ & 0 & 0 & 1 & 1 & 2 & 2 & 0 & 0 & 1 & 1 & 2 & 2 & 0 & 0 & 1 & 1 & 2 & 2 & 0 & 0 & 1 & 1 & 2 \\
\hline $\mathrm{b}$ & 0 & 0 & 0 & 0 & 0 & 0 & 1 & 1 & 1 & 1 & 1 & 1 & 2 & 2 & 2 & 2 & 2 & 2 & 3 & 3 & 3 & 3 & 3 \\
\hline $\mathrm{i}$ & 0 & 1 & 0 & 1 & 0 & 1 & 0 & 1 & 0 & 1 & 0 & 1 & 0 & 1 & 0 & 1 & 0 & 1 & 0 & 1 & 0 & 1 & 0 \\
\hline
\end{tabular}

(a) $m \mapsto(p, b, i)$

\begin{tabular}{|c|rrrrrrrrrrrrrrrrrrrrrrr|r|r|}
\hline $\mathrm{p}$ & 0 & 0 & 0 & 0 & 0 & 0 & 0 & 0 & 1 & 1 & 1 & 1 & 1 & 1 & 1 & 1 & 2 & 2 & 2 & 2 & 2 & 2 & 2 \\
\hline $\mathrm{b}$ & 0 & 0 & 1 & 1 & 2 & 2 & 3 & 3 & 0 & 0 & 1 & 1 & 2 & 2 & 3 & 3 & 0 & 0 & 1 & 1 & 2 & 2 & 3 \\
\hline $\mathrm{i}$ & 0 & 1 & 0 & 1 & 0 & 1 & 0 & 1 & 0 & 1 & 0 & 1 & 0 & 1 & 0 & 1 & 0 & 1 & 0 & 1 & 0 & 1 & 0 \\
\hline $\mathrm{m}$ & 0 & 1 & 6 & 7 & 12 & 13 & 18 & 19 & 2 & 3 & 8 & 9 & 14 & 15 & 20 & 21 & & 5 & 10 & 11 & 16 & 17 & 22 \\
\hline
\end{tabular}

(b) $(p, b, i) \mapsto m$

Figure 9: An example of the block cyclic decomposition of $M=23$ data objects over $P=3$ processes for a block size of $r=2$. (a) shows the mapping from global index, $m$, to the triplet $(p, b, i)$, and (b) shows the inverse mapping.

cyclic decomposition may be written,

$$
m \mapsto\left(\left\lfloor\frac{m \bmod T}{r}\right\rfloor,\left\lfloor\frac{m}{T}\right\rfloor, m \bmod r\right)
$$

where $T=r P$. It should be noted that this reverts to the cyclic decomposition when $r=1$, with local index $i=0$ for all blocks. A block decomposition is recovered when $r=L$, in which case there is a single block in each process with block number $b=0$. The inverse mapping of the triplet $(p, b, i)$ to a global index is given by,

$$
(p, b, i) \mapsto B r+i=p r+b T+i
$$

where $B=p+b P$ is the global block number. The block cyclic decomposition is one of the data distributions supported by High Performance Fortran (HPF) [42], and has been previously used, in one form or another, by several researchers (see $[1,4,5,9,23,27,50,52,54]$ for examples of its use). The block cyclic decomposition is illustrated with an example in Figure 9.

The form of the block cyclic decomposition given by $\mathrm{sq} .11$ ensures that the block with global index 0 is placed in process 0 , the next block is placed in process 1 , and so on. However, it is sometimes necessary to offset the procusses relative to the global block index so that, in general, the first block is placed in process $p_{0}$, the next in process $p_{0}+1$, and so on. We, therefore, generalize the block cyclic decomposition by replacing $m$ on the righthand side of 
Eq. 11 by $m^{\prime}=m+r p_{0}$ to give.

$$
\begin{aligned}
m & \mapsto\left(\left\lfloor\frac{m^{\prime} \bmod T}{r}\right\rfloor,\left\lfloor\frac{m^{\prime}}{T}\right\rfloor, m^{\prime} \bmod r\right) \\
& =\left(\left(\left\lfloor\frac{m \bmod T}{r}\right\rfloor+p_{0}\right) \bmod P_{\cdot}\left\lfloor\frac{m+r p_{0}}{T}\right\rfloor, m \bmod r\right) .
\end{aligned}
$$

Equation 12 may also be generalized to.

$$
(p, b, i) \mapsto B r+i=\left(p-p_{0}\right) r+b T+i
$$

where now the global block number is given by $B=\left(p-p_{0}\right)+b P$. It should be noted that in processes with $p<p_{0}$, block 0 is not within the range of the block cyclic mapping and it is, therefore, an error to reference it in any way.

In decomposing an $M \times N$ matrix we apply independent block cyclic decompositions in the row and column directions. Thus, suppose the matrix rows are distributed with block size $r$ and offset $p_{0}$ over $P$ processes by the block cyclic mapping $\mu_{r, p_{0}, P}$, and the matrix columns are distributed with block size $s$ and offset $q_{0}$ over $Q$ processes by the block cyclic mapping $\nu_{s, q_{0}, Q}$. Then the matrix element indexed globally by $(m, n)$ is mapped as follows,

$$
\begin{aligned}
& m \stackrel{\mu}{\longmapsto}(p, b, i) \\
& n \stackrel{\nu}{\longmapsto}(q, d, j) .
\end{aligned}
$$

The decomposition of the matrix can be regarded as the tensor product of the row and column decompositions, and we can write,

$$
(m, n) \mapsto((p, q),(b, d),(i, j))
$$

The block cyclic matrix decomposition given by Eqs. 15 and 16 distributes blocks of size $r \times s$ to a mesh of $P \times Q$ processes. We shall refer to this mesh as the process template, and refer to processes by their position in the template. Equation 16 says that global index $(m, n)$ is mapped to process $(p, q)$, where it is stored in the block at location $(b, d)$ in a two-iimensional array of blocks. Within this block it is stored at location $(i, j)$. The decomposition is completely specified by the parameters $r, s, p_{0}, q_{0}, P$, and $Q$. In Figure 10 an example is given of the block cyclic decomposition of a $36 \times 80$ matrix for block size $4 \times 5$, a process template $3 \times 4$, and a template offset $\left(p_{0}, q_{0}\right)=(0,0)$. Figure 11 shows the same example but for a template offset of $(1,2)$.

The block cyclic decomposition can reproduce most of the data distributions commonly used in linear algebra computations on parallel computers. For example, if $Q=1$ and $r=\lceil M / P\rceil$ the block row decomposition is obtained. Similarly, $P=1$ and $s=\lceil N / Q\rceil$ gives a block column 
decomposition. These decompositions, together with row and column cyclic decompositions. are shown in Figure 12. Other commonly used block cyclic matrix decompositions are shown in Figure 13.

\section{Parallel Implementation}

In this section we describe the parallel implementation of LU factorization, with partial pivoting over rows, for a block-partitioned matrix. The matrix, $A$, to be factored is assumed to have a block cyclic decomposition, and at the end of the computation is overwritten by the lower and upper triangular factors, $L$ and $U$. This iniplicitly determines the decomposition of $L$ and $U$. Quite a high-level description is given here since the details of the parallel implementation involve optimization issues that will be addressed in Section $\tau$.

The sequential LU factorization algorithm described in Section 4.2 uses square blocks. Although in the parallel algorithm we could choose to decompose the matrix using nonsquare blocks, this would result in a more complicated code, and additional sources of concurrent overhead. For LU factorization we, therefore, restrict the decomposition to use only square blocks, so that the blocks used to decompose the matrix are the same as those used to partition the computation. If the block size is $r \times r$, then an $M \times N$ matrix consists of $M_{b} \times N_{b}$ blocks, where $M_{b}=\lceil M / r\rceil$ and $N_{b}=\lceil N / r\rceil$.

As discussed in Section 4.2, LU factorization proceeds in a series of sequential steps indexed by $k=0, \min \left(M_{b}, N_{b}\right)-1$, in each of which the following three tasks are performed,

1. factor the $k$ th column of blocks, performing pivoting if necessary. This evaluates the matrices $L_{0}, L_{1}$, and $U_{0}$ in Figure 6.

2. evaluate the $k$ th block row of $U$ by solving the lower triangular system $L_{0} U_{1}=C$.

3. do a rank- $r$ update on the trailing submatrix $E$, replacing it with $E^{\prime}=E-L_{1} U_{1}$.

We now consider the parallel implementation of each of these tasks. The computation in the factorization step involves a single column of blocks, and these lie in a single column of the process template. In the $k$ th factorization step, each of the $r$ columns in block column $k$ is processed in turn. Consider the ith column in block column $k$. The pivot is selected by finding the element with largest absolute value in this column between row $k r+i$ and the last row, inclusive. The elements involved in the pivot search at this stage are shown shaded in Figure 14. Having selected the pivot, the value of the pivot and its row are broadcast to all other processors. Next, pivoting is performed by exchanging the entire row $k r+i$ with the row containing the pivot. We exchange entire rows, rather than just the part to the right of the columns already factored, in order to simplify the application of the pivots to the righthand side in any subsequent solve phase. Finally, each value in the column below the pivot is divided by 


\begin{tabular}{r|c|c|c|c|c|c|c|c|c|c|c|c|c|c|c|c|c|c|}
\hline$p, q$ & 0 & 1 & 2 & 3 & 4 & 5 & 6 & 7 & 8 & 9 & 10 & 11 & 12 & 13 & 14 & 15 \\
\hline 0 & 0,0 & 0,1 & 0,2 & 0,3 & 0,0 & 0,1 & 0,2 & 0,3 & 0,0 & 0,1 & 0,2 & 0,3 & 0,0 & 0,1 & 0,2 & 0,3 \\
\hline 1 & 1,0 & 1,1 & 1,2 & 1,3 & 1,0 & 1,1 & 1,2 & 1,3 & 10 & 1,1 & 1,2 & 1,3 & 1,0 & 1,1 & 1,2 & 1,3 \\
\hline 2 & 2,0 & 2,1 & 2,2 & 2,3 & 2,0 & 2,1 & 2,2 & 2,3 & 2,0 & 2,1 & 2,2 & 2,3 & 2,0 & 2,1 & 2,2 & 2,3 \\
\hline 3 & 0,0 & 0,1 & 0,2 & 0,3 & 0,0 & 0,1 & 0,2 & 0,3 & 0,0 & 0,4 & 0,2 & 0,3 & 0,0 & 0,1 & 0,2 & 0,3 \\
\hline 4 & 1,0 & 1,1 & 1,2 & 1,3 & 1,0 & 1,1 & 1,2 & 1,3 & 1,0 & 1,1 & 1,2 & 1,3 & 1,0 & 1,1 & 1,2 & 1,3 \\
\hline 5 & 2,0 & 2,1 & 2,2 & 2,3 & 2,0 & 2,1 & 2,2 & 2,3 & 2,0 & 2,1 & 2,2 & 2,3 & 2,0 & 2,1 & 2,2 & 2,3 \\
\hline 6 & 0,0 & 0,1 & 0,2 & 0,3 & 0,0 & 0,1 & 0,2 & 0,3 & 0,0 & 0,1 & 0,2 & 0,3 & 0,0 & 0,1 & 0,2 & 0,3 \\
7 & 1,0 & 1,1 & 1,2 & 1,3 & 1,0 & 1,1 & 1,2 & 1,3 & 1,0 & 1,1 & 1,2 & 1,3 & 1,0 & 1,1 & 1,2 & 1,3 \\
\hline 8 & 2,0 & 2,1 & 2,2 & 2,3 & 2,0 & 2,1 & 2,2 & 2,3 & 2,0 & 2,1 & 2,2 & 2,3 & 2,0 & 2,1 & 2,2 & 2,3 \\
9 & 0,0 & 0,1 & 0,2 & 0,3 & 0,0 & 0,1 & 0,2 & 0,3 & 0,0 & 0,1 & 0,2 & 0,3 & 0,0 & 0,1 & 0,2 & 0,3 \\
10 & 1,0 & 1,1 & 1,2 & 1,3 & 1,0 & 1,1 & 1,2 & 1,3 & 1,0 & 1,1 & $\$, 2$ & 1,3 & 1,0 & 1,1 & 1,2 & 1,3 \\
\hline 11 & 2,0 & 2,1 & 2,2 & 2,3 & 2,0 & 2,1 & 2,2 & 2,3 & 2,0 & 2,1 & 2,2 & 2,3 & 2,0 & 2,1 & 2,2 & 2,3 \\
\hline
\end{tabular}

(a) Assignment of global block indices, $(B, D)$, to processes, $(p, q)$.

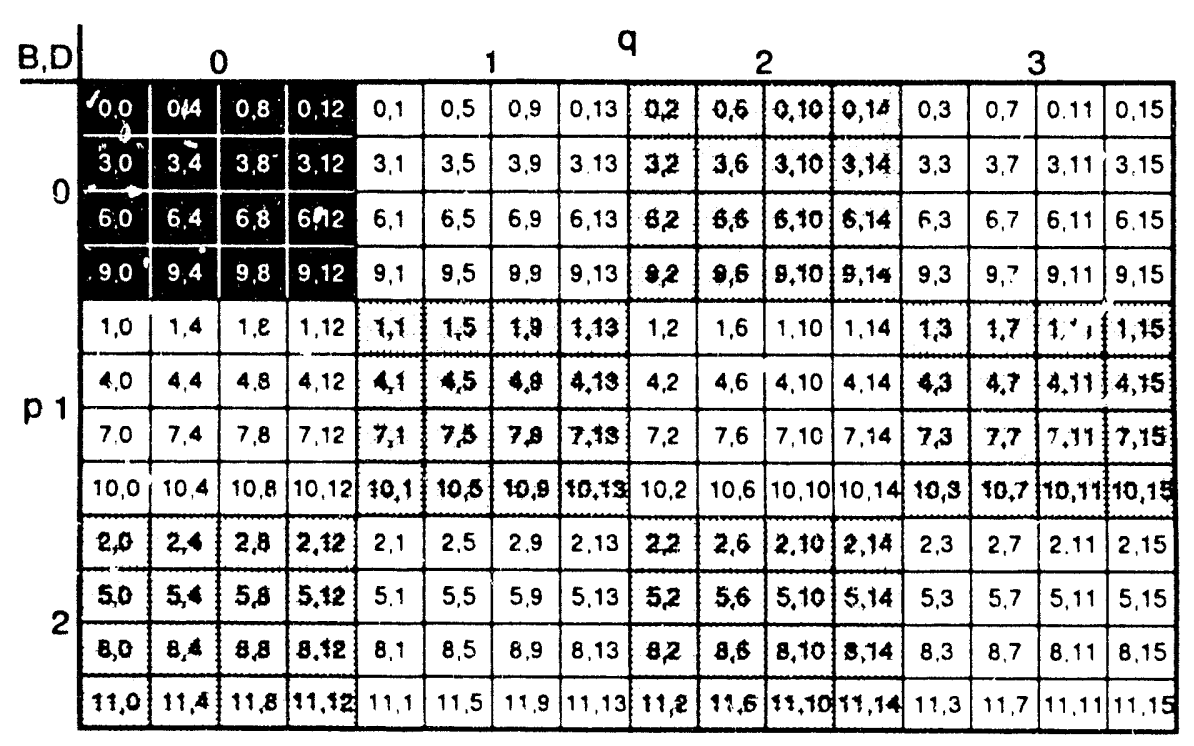

(b) Cobal blocks. (B, D) , in each process, $(p, q)$

1 equre 10 Block ryche decomposition of a $36 \times 80$ matrix with a block size of 4 , 5. onto a 3. A process template Each small rectangle represents onf matrix block indivdual matrix elements are not shown In (a), shading is used to emphasize the process emplite that is permdically stamped over the matrix. and each block is labeled with the process to which it is assignt dn (b) each shaded region shows the blocks in one process, and is labeled wnh the corresponding global block indices. In buth figures. the bli k rectangles indicate the blocks assigned to process $(0,0)$ 


\begin{tabular}{|c|c|c|c|c|c|c|c|c|c|c|c|c|c|c|c|c|}
\hline q & 0 & 1 & 2 & 3 & 4 & 5 & 6 & 7 & 8 & 9 & 10 & 11 & 12 & 13 & 4 & 15 \\
\hline 0 & 1,2 & 1,3 & 1.0 & 17 & 1,2 & 1,3 & 1,0 & 1,1 & 1,2 & 1,3 & 1,0 & 1,1 & 2 & 3 & .0 & 1 \\
\hline 1 & 2.2 & 2,3 & 2,0 & 2.1 & 2,2 & 2 & 2.0 & 2,1 & 2,2 & 23 & $a r$ & 31 & 2,2 & 3 & 2,0 & 21 \\
\hline 2 & 0,2 & 0,3 & 0,0 & 0.1 & 0,2 & 0,3 & 0,0 & 0,1 & 0,2 & 0,3 & 0,0 & 0.1 & 0,2 & .3 & 0 & 0.1 \\
\hline 3 &, \pm 2 & 1,3 & 1,0 & 1.1 & 1,2 & 1,3 & 1,0 & $1+1$ & 1,2 & 1,5 & 1,0 & 1,1 & 1,2 & 1,3 & 1,0 & 1,1 \\
\hline 4 & 2,2 & 2,3 & .0 & 21 & 2,2 & 2,3 & 2,0 & 2,1 & 22 & 2,3 & 2,0 & 2,1 & 2,2 & 2,3 & 2,0 & 2,1 \\
\hline & 0,2 & 0,3 & 0,0 & 0,1 & 0,2 & 0,3 & 0,0 & 0.1 & 0,2 & 0,3 & 0,0 & 0 & 0.2 & 0,3 &, 0 & 0,1 \\
\hline & 1,2 & 1,3 & 1.0 & 1,1 & 12 & 13 & 1,0 & 1,1 & 1,2 & 1,3 & 10 & 11 & 1,2 & 1,3 & 1,0 & 1,1 \\
\hline 7 & 2,2 & 2,3 & 2,0 & 2,1 & 2,2 & 2,3 & 2,0 & 2.1 & 2,2 & 2,3 & 2,0 & 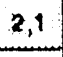 & 2,2 & 2,3 & 2,0 & 2.1 \\
\hline 8 & 0,2 & 0,3 & 0,0 & 0,1 & 0,2 & 0,3 & 0,0 & 0,1 & 0 & 0,3 & 0,0 & 0,1 & 0.2 & 0,3 & 0,0 & 0.1 \\
\hline 9 & 1,2 & 1,5 & נ, ני & 1,1 & 1,2 & 1,3 & 1,0 & $x_{x}$ & 1,2 & 1,3 & 1,0 & 1.1 & 1,2 & 1,3 & 10 & 1,1 \\
\hline 10 & 2,2 & 2,3 & 2,0 & 2,1 & 2,2 & 2,3 & 2,0 & 2,1 & 2,2 & 2,3 & 2,0 & 2,1 & 2 & 2,3 & 20 & 2, \\
\hline 11 & 0,2 & 0,3 & 0,0 & 0,7 & 0,2 & 0,3 & 0,0 & 0.1 & 0,2 & 0 & 0,0 & 1 & 0,2 & 0,3 & 0,0 & 0,1 \\
\hline
\end{tabular}

(a) Assignment of global block indices, $(B, D)$, to processes, $(p, q)$.

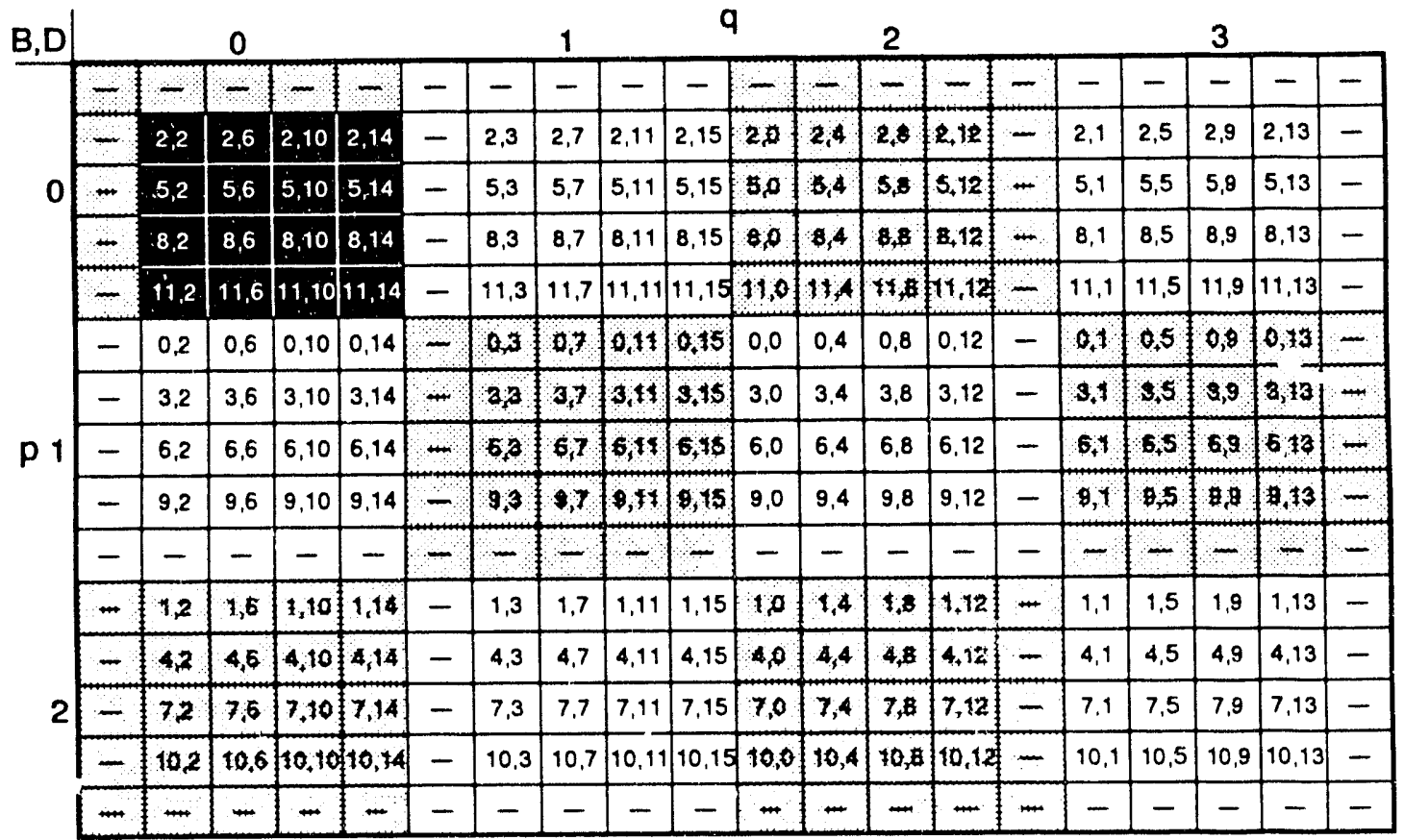

(b) Global blocks, $(B, D)$, in each process. $(p, q)$

Figure 11: The same matrix decomposition as shown in Figure 10. but for a template offset of $\left(p_{0}, q_{0}\right)=(1,2)$. Dashed entries in (b) indicate that the block does not contain any data. In both figures, the blacl: rectangles indicate the blocks assigned to process $(0,0)$. 


\begin{tabular}{|l|l|l|l|l|l|l|l|l|l|l|}
\hline 0,0 & 0,0 & 0,0 & 0,0 & 0,0 & 0,0 & 0,0 & 0,0 & 0,0 & 0,0 \\
\hline 0,0 & 0,0 & 0,0 & 0,0 & 0,0 & 0,0 & 0,0 & 0,0 & 0,0 & 0,0 \\
\hline 0,0 & 0,0 & 0,0 & 0,0 & 0,0 & 0,0 & 0,0 & 0,0 & 0,0 & 0,0 \\
\hline 1,0 & 1,0 & 1,0 & 1,0 & 1,0 & 1,0 & 1,0 & 1,0 & 1,0 & 1,0 \\
\hline 1,0 & 1,0 & 1,0 & 1,0 & 1,0 & 1,0 & 1,0 & 1,0 & 1,0 & 1,0 \\
\hline 1,0 & 1,0 & 1,0 & 1,0 & 1,0 & 1,0 & 1,0 & 1,0 & 1,0 & 1,0 \\
\hline 2,0 & 2,0 & 2,0 & 2,0 & 2,0 & 2,0 & 2,0 & 2,0 & 2,0 & 2,0 \\
\hline 2,0 & 2,0 & 2,0 & 2,0 & 2,0 & 2,0 & 2,0 & 2,0 & 2,0 & 2,0 \\
\hline 2,0 & 2,0 & 2,0 & 2,0 & 2,0 & 2,0 & 2,0 & 2,0 & 2,0 & 2,0 \\
\hline 3,0 & 3,0 & 3,0 & 3,0 & 3,0 & 3,0 & 3,0 & 3,0 & 3,0 & 3,0 \\
\hline
\end{tabular}

(a) $r=3, s=10, P=4, Q=1$

\begin{tabular}{|l|l|l|l|l|l|l|l|l|l|}
\hline 0,0 & 0,0 & 0,0 & 0,1 & 0,1 & 0,1 & 0,2 & 0,2 & 0,2 & 0,3 \\
\hline
\end{tabular}

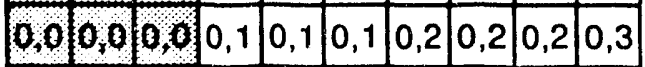

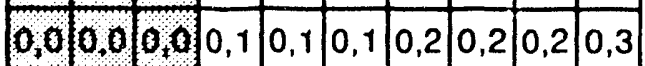

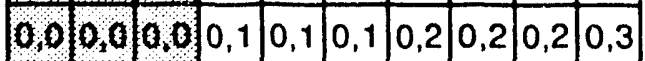
\begin{tabular}{ll|l|l|l|l|l|l|l|l|}
\hline 0,0 & 0,0 & 0,0 & 0,1 & 0,1 & 0,1 & 0,2 & 0,2 & 0,2 & 0,3 \\
\hline
\end{tabular}

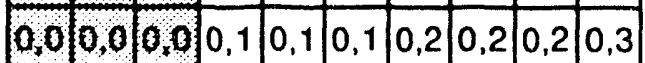

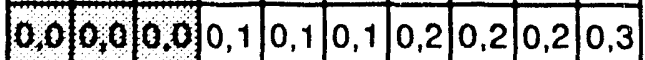
\begin{tabular}{lllllllllll}
0,0 & 0,0 & 0,0 & 0,1 & 0,1 & 0,1 & 0,2 & 0,2 & 0,2 & 0,3 \\
\hline
\end{tabular} \begin{tabular}{llllllllllll|}
\hline 0,0 & 0,0 & 0,0 & 0,1 & 0,1 & 0,1 & 0,2 & 0,2 & 0,2 & 0,3 \\
\hline
\end{tabular}

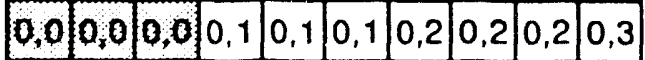
(c) $r=10 . s=3, P=1, Q=4$

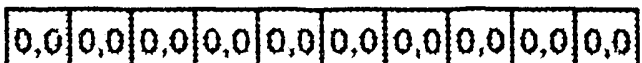
\begin{tabular}{|l|l|l|l|l|l|l|l|l|l|}
\hline 1,0 & 1,0 & 1,0 & 1,0 & 1,0 & 1,0 & 1,0 & 1,0 & 1,0 & 1,0 \\
\hline
\end{tabular} \begin{tabular}{|l|l|l|l|l|l|l|l|l|l|}
\hline 2,0 & 2,0 & 2,0 & 2,0 & 2,0 & 2,0 & 2,0 & 2,0 & 2,0 & 2,0 \\
\hline
\end{tabular} \begin{tabular}{|l|l|l|l|l|l|l|l|l|l|}
\hline 3,0 & 3,0 & 3,0 & 3,0 & 3,0 & 3,0 & 3,0 & 3,0 & 3,0 & 3,0 \\
\hline
\end{tabular} \begin{tabular}{lllllllllll}
\hline 0,0 & 0,0 & 0,0 & 0,0 & 0,0 & 0,0 & 0,0 & 0,0 & 0,0 & 0,0 \\
\hline
\end{tabular} \begin{tabular}{|l|l|l|l|l|l|l|l|l|l|}
1,0 & 1,0 & 1,0 & 1,0 & 1,0 & 1,0 & 1,0 & 1,0 & 1,0 & 1,0 \\
\hline
\end{tabular} \begin{tabular}{|l|l|l|l|l|l|l|l|l|l|}
\hline 2,0 & 2,0 & 2,0 & 2,0 & 2,0 & 2,0 & 2,0 & 2,0 & 2,0 & 2,0 \\
\hline
\end{tabular} \begin{tabular}{|l|l|l|l|l|l|l|l|l|l|}
\hline 3,0 & 3,0 & 3,0 & 3,0 & 3,0 & 3,0 & 3,0 & 3,0 & 3,0 & 3,0 \\
\hline
\end{tabular}

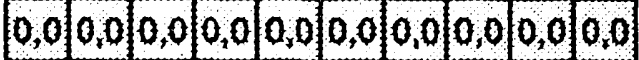
\begin{tabular}{|l|l|l|l|l|l|l|l|l|l|}
\hline 1,0 & 1,0 & 1,0 & 1,0 & 1,0 & 1,0 & 1,0 & 1,0 & 1,0 & 1,0 \\
\hline
\end{tabular}

(b) $r=1, s=10 . P=4, Q=1$

\begin{tabular}{|l|l|l|l|l|l|l|l|l|l|}
\hline 0,0 & 0,1 & 0,2 & 0,3 & 0,0 & 0,1 & 0,2 & 0,3 & 0,0 & 0,1 \\
\hline
\end{tabular} \begin{tabular}{lllllllllllll}
0,0 & 0,1 & 0,2 & 0,3 & 0,0 & 0,1 & 0,2 & 0,3 & 0,0 & 0,1 \\
\hline
\end{tabular} \begin{tabular}{lllllllllll}
\hline 0,0 & 0,1 & 0,2 & 0,3 & 0,0 & 0,1 & 0,2 & 0,3 & 0,0 & 0,1 \\
\hline
\end{tabular} \begin{tabular}{llllllllllll}
0,0 & 0,1 & 0,2 & 0,3 & 0,0 & 0,1 & 0,2 & 0,3 & 0,0 & 0,1 \\
\hline
\end{tabular} \begin{tabular}{llllllllllll}
$0,0,1$ & 0,2 & 0,3 & 0,0 & 0,1 & 0,2 & 0,3 & 0,0 & 0,1 \\
\hline
\end{tabular} \begin{tabular}{llllllllllll}
0,0 & 0,1 & 0,2 & 0,3 & 0,0 & 0,1 & 0,2 & 0,3 & 0,0 & 0,1 \\
\hline
\end{tabular} \begin{tabular}{llllllllllll}
0,0 & 0,1 & 0,2 & 0,3 & 0,0 & 0,1 & 0,2 & 0,3 & 0,0 & 0,1 \\
\hline
\end{tabular} \begin{tabular}{llllllllllll}
0,0 & 0,1 & 0,2 & 0,3 & 0,0 & 0,1 & 0,2 & 0,3 & 0,0 & 0,1 \\
\hline
\end{tabular} \begin{tabular}{lllllllllllll}
0,0 & 0,1 & 0,2 & 0,3 & 0,0 & 0,1 & 0,2 & 0,3 & 0,0 & 0,1 \\
\hline
\end{tabular}

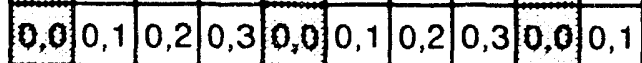

(d) $r=11, \cdot r=1, P^{\prime}=1 \cdot Q=4$

Figure 12: These 4 figures show different ways of decomposing it 10, 10 matrix. Each cell

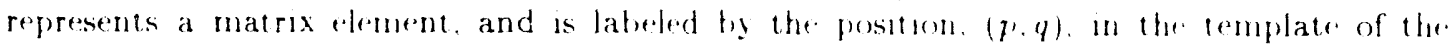
process to which it is assigned. 'Io emptasize the pattern of decomposition. the matrix entree assigned to the process in the first row and column of the template are shown shaded. and each separate shaded region represents a matrix bloch. Figures (a) and (b) show hlock and cyclie row-oriented decompositions. respectively for 4 nodes. In figures (c) and (d) the corresponding column-oriented decompositions are shown. Below each figure we give the values of $r$. s. $P$. and $Q$ corresponding to the decomposition In all cases $p_{0}=y_{i 1}=0$. 


\begin{tabular}{|l|l|l|l|l|l|l|l|l|l|}
\hline $0,010,0$ & 0,0 & 0,1 & 0,1 & 0,1 & 0,2 & 0,2 & 0,2 & 0,3 \\
\hline 0,0 & 0,0 & 0,0 & 0,1 & 0,1 & 0,1 & 0,2 & 0,2 & 0,2 & 0,3 \\
\hline 0,0 & 0,0 & 0,0 & 0,1 & 0,1 & 0,1 & 0,2 & 0,2 & 0,2 & 0,3 \\
\hline 1,0 & 1,0 & 1,0 & 1,1 & 1,1 & 1,1 & 1,2 & 1,2 & 1,2 & 1,3 \\
\hline 1,0 & 1,0 & 1,0 & 1,1 & 1,1 & 1,1 & 1,2 & 1,2 & 1,2 & 1,3 \\
\hline 1,0 & 1,0 & 1,0 & 1,1 & 1,1 & 1,1 & 1,2 & 1,2 & 1,2 & 1,3 \\
\hline 2,0 & 2,0 & 2,0 & 2,1 & 2,1 & 2,1 & 2,2 & 2,2 & 2,2 & 2,3 \\
\hline 2,0 & 2,0 & 2,0 & 2,1 & 2,1 & 2,1 & 2,2 & 2,2 & 2,2 & 2,3 \\
\hline 2,0 & 2,0 & 2,0 & 2,1 & 2,1 & 2,1 & 2,2 & 2,2 & 2,2 & 2,3 \\
\hline 3,0 & 3,0 & 3,0 & 3,1 & 3,1 & 3,1 & 3,2 & 3,2 & 3,2 & 3,3 \\
\hline
\end{tabular}

(a) $r=3, s=3, P=4, Q=4$

\begin{tabular}{|l|l|l|l|l|l|l|l|l|l|}
\hline 3. & $0.0,1$ & 0,1 & 0,1 & 0,2 & 0,2 & 0,2 & 0,3 \\
\hline 1,0 & 1,0 & 1,0 & 1,1 & 1,1 & 1,1 & 1,2 & 1,2 & 1,2 & 1,3 \\
\hline 2,0 & 2,0 & 2,0 & 2,1 & 2,1 & 2,1 & 2,2 & 2,2 & 2,2 & 2,3 \\
\hline 3,0 & 3,0 & 3,0 & 3,1 & 3,1 & 3,1 & 3,2 & 3,2 & 3,2 & 3,3 \\
\hline 0 & & 0 & 0,1 & 0,1 & 0,1 & 0,2 & 0,2 & 0,2 & 0,3 \\
\hline 1,0 & 1,0 & 1,0 & 1,1 & 1,1 & 1,1 & 1,2 & 1,2 & 1,2 & 1,3 \\
\hline 2,0 & 2,0 & 2,0 & 2,1 & 2,1 & 2,1 & 2,2 & 2,2 & 2,2 & 2,3 \\
\hline 3,0 & 3,0 & 3,0 & 3,1 & 3,1 & 3,1 & 3,2 & 3,2 & 3,2 & 3,3 \\
\hline 0,0 & 0,0 & 0,0 & 0,1 & 0,1 & 0,1 & 0,2 & 0,2 & 0,2 & 0,3 \\
\hline 1,0 & 1,0 & 1,0 & 1,1 & 1,1 & 1,1 & 1,2 & 1,2 & 1,2 & 1,3 \\
\hline
\end{tabular}

(c) $r=1, s=3, P=4, Q=4$

\begin{tabular}{|l|l|l|l|l|l|l|l|l|l|}
\hline 0,0 & 0,1 & 0,2 & 0,3 & 0,0 & 0,1 & 0,2 & 0,3 & 0,0 & 0,1 \\
\hline 0,0 & 0,1 & 0,2 & 0,3 & 0,0 & 0,1 & 0,2 & 0,3 & 0,0 & 0,1 \\
\hline 0,0 & 0,1 & 0,2 & 0,3 & 0,0 & 0,1 & 0,2 & 0,3 & 0,0 & 0,1 \\
\hline 1,0 & 1,1 & 1,2 & 1,3 & 1,0 & 1,1 & 1,2 & 1,3 & 1,0 & 1,1 \\
\hline 1,0 & 1,1 & 1,2 & 1,3 & 1,0 & 1,1 & 1,2 & 1,3 & 1,0 & 1,1 \\
\hline 1,0 & 1,1 & 1,2 & 1,3 & 1,0 & 1,1 & 1,2 & 1,3 & 1,0 & 1,1 \\
\hline 2,0 & 2,1 & 2,2 & 2,3 & 2,0 & 2,1 & 2,2 & 2,3 & 2,0 & 2,1 \\
\hline 2,0 & 2,1 & 2,2 & 2,3 & 2,0 & 2,1 & 2,2 & 2,3 & 2,0 & 2,1 \\
\hline 2,0 & 2,1 & 2,2 & 2,3 & 2,0 & 2,1 & 2,2 & 2,3 & 2,0 & 2,1 \\
\hline 3,0 & 3,1 & 3,2 & 3,3 & 3,0 & 3,1 & 3,2 & 3,3 & 3,0 & 3,1 \\
\hline
\end{tabular}

(b) $r=3, s=1, P=4, Q=4$

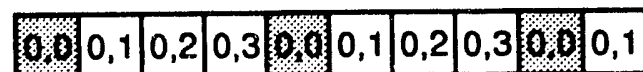
\begin{tabular}{|l|l|l|l|l|l|l|l|l|l|}
\hline 1,0 & 1,1 & 1,2 & 1,3 & 1,0 & 1,1 & 1,2 & 1,3 & 1,0 & 1,1 \\
\hline
\end{tabular} \begin{tabular}{|l|l|l|l|l|l|l|l|l|l|}
\hline 2,0 & 2,1 & 2,2 & 2,3 & 2,0 & 2,1 & 2,2 & 2,3 & 2,0 & 2,1 \\
\hline
\end{tabular} \begin{tabular}{|l|l|l|l|l|l|l|l|l|l|}
\hline 3,0 & 3,1 & 3,2 & 3,3 & 3,0 & 3,1 & 3,2 & 3,3 & 3,0 & 3,1 \\
\hline
\end{tabular}

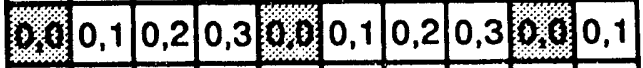
\begin{tabular}{|l|l|l|l|l|l|l|l|l|l|}
\hline 1,0 & 1,1 & 1,2 & 1,3 & 1,0 & 1,1 & 1,2 & 1,3 & 1,0 & 1,1 \\
\hline
\end{tabular} \begin{tabular}{|l|l|l|l|l|l|l|l|l|l|}
\hline 2,0 & 2,1 & 2,2 & 2,3 & 2,0 & 2,1 & 2,2 & 2,3 & 2,0 & 2,1 \\
\hline
\end{tabular} \begin{tabular}{|l|l|l|l|l|l|l|l|l|l|}
\hline 3,0 & 3,1 & 3,2 & 3,3 & 3,0 & 3,1 & 3,2 & 3,3 & 3,0 & 3,1 \\
\hline
\end{tabular}

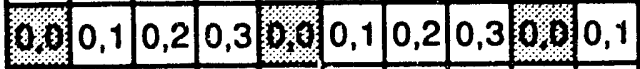
\begin{tabular}{|l|l|l|l|l|l|l|l|l|l|}
\hline 1,0 & 1,1 & 1,2 & 1,3 & 1,0 & 1,1 & 1,2 & 1,3 & 1,0 & 1,1 \\
\hline
\end{tabular}

(d) $r=1, s=1, P=4, Q=4$

Figure 13: These 4 figures show different ways of decomposing a $10 \times 10$ matrix over 16 processes arranged as a $4 \times 4$ template. Below each figure we give the values of $r, s, P$, and $Q$ corresponding to the decomposition. In all cases $p_{0}=q_{0}=0$. 


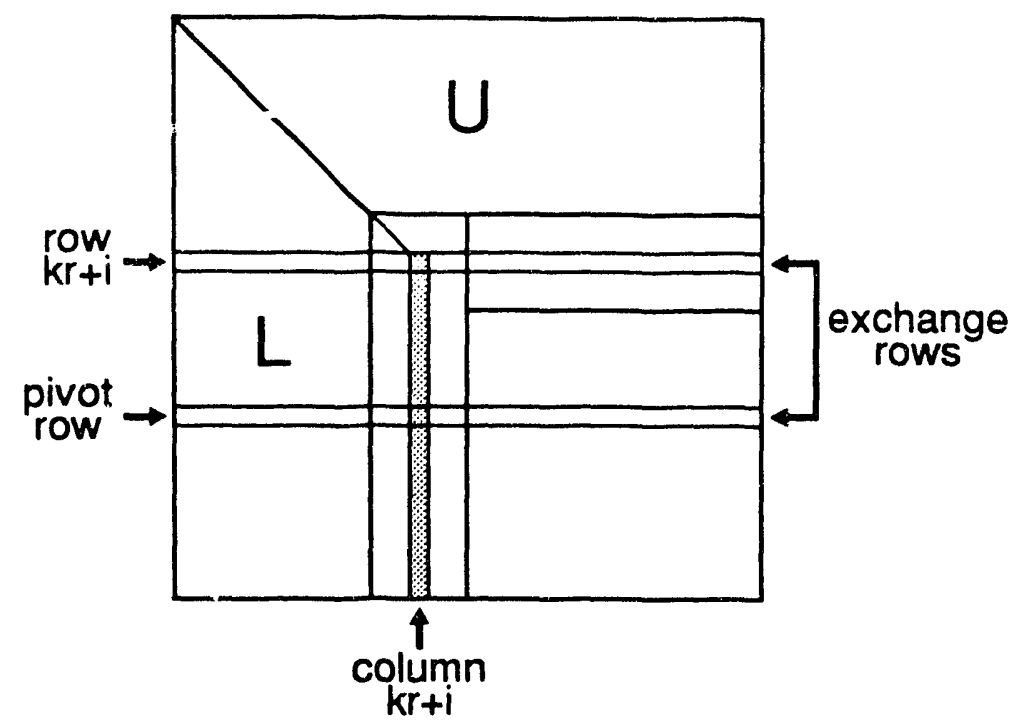

Figure 14: This figure shows pivoting for step $i$ of the $k$ th stage of $L U$ factorization. The element with largest absolute value in the gray shaded part of column $k r+i$ is found, and the row containing it is exchanged with row $k r+i$. If the rows exchanged lie in different processes, communication may be necessary.

the pivot. If a cyclic column decomposition is used, like that shown in Figure 12(d), only one processor is involved in the factorization of the block column, and no communication is necessary between the processes. However, in general $P$ processes are involved, and communication is necessary in selecting the pivot, and exchanging the pivot rows.

The solution of the lower triangular system $L_{0} U_{1}-C$ to evaluate the kth block row of $U$ involves a single row of blocks, and these lie in a single row of the process template. If a cyclic row decomposition is used, like that shown in Figure 12(b), only one processor is involved in the triangular solve, and no communication is necessary between the processes. However, in general $Q$ processes are involved, and communication is necessary to broadcast the lower triangular matrix, $L_{0}$, to all processes in the row. Once this has been done, each process in the row independently performs a lower triangular solve for the blocks of $C$ that it holds.

The communication necessary to update the trailing submatrix at step $k$ takes place in two steps. First, each process holding part of $L_{1}$ broadcasts these blocks to the other processes in the same row of the template. This may be done in conjunction with the broadcast of $L_{0}$, mentioned in the preceding paragraph, so that all of the factored panel is broadcast together. Next, each process holding part. of $U_{1}$ broadcasts these blocks to the other processes in the same column of the template. Each process can then complete the update of the blocks that it holds with no further communication.

A pseudocode outline of the parallel $L U$ factorization algorithm is given in Figure 15. There 


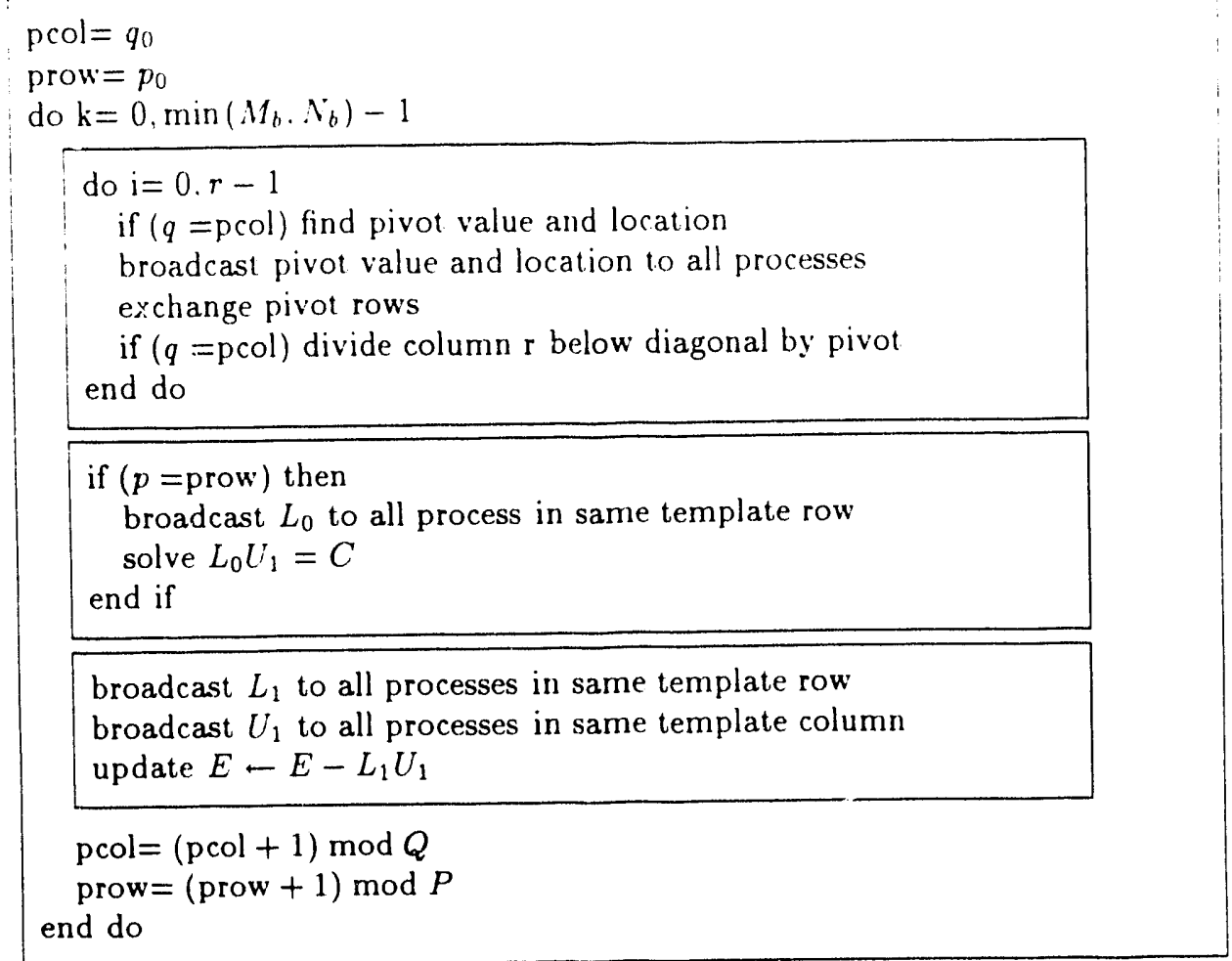

Figure 15: Pseudocode for the basic parallel block-partitioned LU factorization algorithm. This code is executed by each process. The first box inside the $k$ loop factors the $k$ th column of blocks. The second box solves a lower triangular system to evaluate the $k$ th row of blocks of $U$, and the third box updates the trailing submatrix. The template offset is given by $\left(p_{\iota} q_{0}\right)$, and $(p, q)$ is position of a process in the template.

are two points worth noting in Figure 15. First, the triangular solve and update phases operate on matrix blocks and may, therefore, be done with parallel versions of the Level 3 BLAS (specifically, xTRSM and xGEMM, respectively). The factorization of the column of blocks, however, involves a loop over matrix columns. Hence, is it not a block-oriented computation, and cannot be performed using the Level 3 BLAS. The second point to note is that most of the parallelism in the code comes from updating the trailing submatrix since this is the only phase in which all the processes are busy.

Figure 15 also shows quite clearly where communication is required; namely, in finding the pivot, exchanging pivot rows, and performing various types of broadcast. The exact way in which these communications are done and interleaved with computation generally has an important effect on performance, and will be discussed in more detail in Section 7 .

Figure 15 refers to broadcasting data to all processes in the same row or column of the template. This is a common operation in parallel linear algebra algorithms, so the idea will be described here in a little more detail. Consider, for example, the task of broadcasting the 


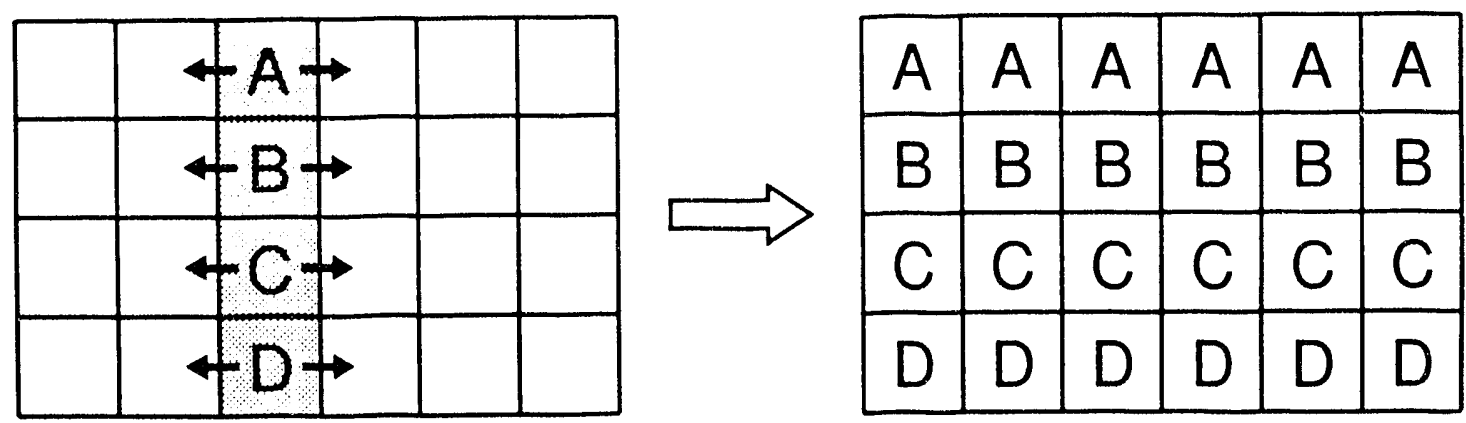

(a) Broadcast along rows.
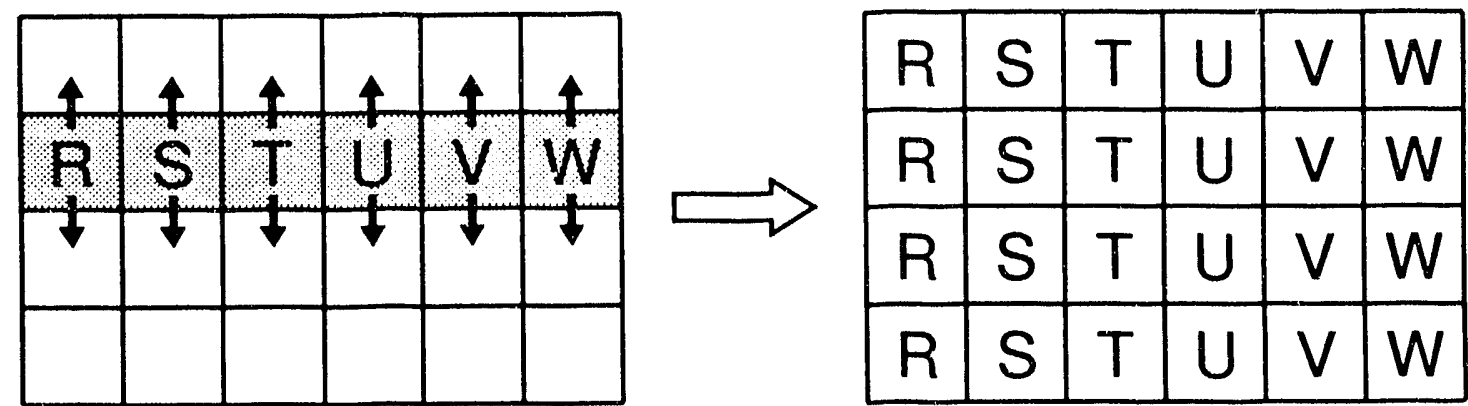

(b) Broadcast along columns.

Figure 16: Schematic representation of broadcast along rows and columns of a $4 \times 6$ process template. In (a), each shaded process broadcasts to the processes in the same row of the process template. In (b), each shaded process broadcasts to the processes in the same column of the process template.

lower triangular block, $L_{0}$, to all processes in the same row of the template, as required before solving $L_{0} U_{1}=C$. If $L_{0}$ is in process $(p, q)$, then it will be broadcast to all processes in row $p$ of the process template. As a second example, consider the broadcast of $L_{1}$ to all processes in the same template row, as required before updating the trailing submatrix. This type of "rowcast" is shown schematically in Figure 16(a). If $L_{1}$ is in column $q$ of the template, then each process $(p, q)$ broadcasts its blocks of $L_{1}$ to the other processes in row $p$ of the template Loosely speaking, we can say that $L_{0}$ and $L_{1}$ are broadcast along the rows of the template This type of data movement is the same as that performed by the Fortran 90 routine SPREAD [7]. The broadcast of $U_{1}$ to all processes in the same template column is very similar. This type of communication is sometimes referred to as a "colcast", and is shown in Figure 16(b). 


\section{Optimization, Tuning, and Trade-offs}

In this section, we shall examine techniques for optimizing the basic Ll factorization code presented in Section 4.2. Among the issues to be considered are the assignment of processes to physical processors, the arrangement of the data in the local memory of each process, the trade-off between load imbalance and communication latency, the potential for overlapping communication and calculation, and the type of algorithm used to broadcast data. Many of these issues are interdependent, and in addition the portability and ease of code maintenance and use must be considered. For further details of the optimization of parallel LU factorization algorithms for specific concurrent machines, together with timing results, the reader is referred to the work of Chu and George [12], Geist and Heath [34], Geist and Romine [35], Van de Velde [55], Brent [8], Hendrickson and Womble [39], Lichtenstein and Johnsson [47], and Dongarra and co-workers $[10,25]$.

\subsection{Mapping Logical Memory to Physical Memory}

In Sertion 5, a logical (or virtual) matrix decomposition was described in which the global index $(m, n)$ is mapped to a position, $(p, q)$, in a logical process template, a position, $(b, d)$, in a logical array of blocks local to the process, and a position, $(i, j)$, in a logical array of matrix elements local to the block. Thus, the block cyclic decomposition is hierarchical, and attempts to represent the hierarchical memory of advanced-architecture computers. Although the parallel LU factorization algorithm can be specified solely in terms of this logical hierarchical memory, its performance depends on how the logical memory is mapped to physical memory.

\subsubsection{Assignment of Processes to Processors}

Consider, first, the assignment of processes, $(p, q)$, to physical processors. In general, more than one process may be assigned to a processor, so the problem may be overdecomposed. To avoid load imbalance the same number of processes should be assigned to each processor as nearly as possible. If this condition is satisfied, the assignment of processes to processors can still affect performance by influencing the communication overhead. On recent distributed memory machines, such as the Intel Delta and CM-5, the time to send a single message between two processors is largely independent of their physical location $[29,48,49]$, and hence the assignment of processes to processors does not have much direct effect on performance. However, when a collective communication task, such as a broadcast, is being done, contention for physical resources can degrade performance. Thus, the way in which processes are assigned to processors can affect performance if some assignments result in differing amounts of contention. Logarithmic contention-free broadcast algorithms have been developed for processors connected as a two-dimensional mesh $[6,51]$, so on such machines process $(p, q)$ is usually mapped to the 
processor at position $(\mu, q)$ in the mesh of processors. Such an assignment also ensures that the multiple one-dimensional broadeasts of $L_{1}$ and $l_{1}$ along the rows and columns of the template respectively, do not give rise' to contention.

\subsubsection{Layout of Local Process Memory}

The layout of matrix blocks in the local memory of a process, and the arrangement of matrix elements within each block, can also affect performance. Here, tradeoffs among several factors need to be taken into account. When communicating matrix blocks, for example in the broadcasts of $L_{1}$ and $U_{1}$, we would like the data in each block to be contiguous in physical memory so there is no need to pack them into a communication buffer before sending them. On the other hand, when updating the trailing submatrix, $E$, each process multiplies a column of blocks by a row of blocks, to do a rank-r update on the part of $E$ that it contains. If this were done as a series of separate block-block matrix multiplications, as shown in Figure 18(a), the performance would be poor except for sufficiently large block sizes, $r$, since the vector and/or pipeline units on most processors would not be fully utilized, as may be seen in Figure 17 for the i860 processor. Instead, we arrange the loops of the computation as shown in Figure 18(b). Now, if the data are laid out in physical memory first by running over the $i$ index and then over the $d$ index the inner two loops can be merged, so that the length of the inner loop is now $r d_{\max }$. This generally results in much better vector/pipeline performance. The $b$ and $j$ loops in Figure 18(b) can also be merged, giving the algorithm shown in Figure 18(c). This is just the outer product form of the multiplication of an $r d_{\max } \times r$ by an $r \times r b_{\max } \operatorname{matrix}$, and would usually be done by a call to the Level 3 BLAS routine XGEMM of which an assembly coded sequential version is avaiable on most machines. Note that in Figure 18(c) the order of the inner two loops is appropriate for a Fortran implementation - for the C language this order should be reversed, and the data should be stored in each process by rows instead of by columns.

We have found in our work on the Intel iPSC/860 hypercube and the Delta system that. it is better to optimize for the sequential matrix multiplication with an $(i, d, j, b)$ ordering of mernory in each process, rather than adopting an $(i, j, d, b)$ ordering to avoid buffer copites when communicating blocks. However, there is another reason for doing this. On most distributed memory computers the message startup cost is sufficiently large what it is preferable wherever possible to send data as one large message rather than as several smaller messages. Thus, when communicating $L_{1}$ and $U_{1}$ the blocks to be broadcast would be amalgamated into a single message, which requires a buffer copy. The emerging Message Passing Interface (MPI) standard [21] provides support for noncontiguous messages, so in the future the need to avoid buffer copies will not be of such concern to the application developer. 


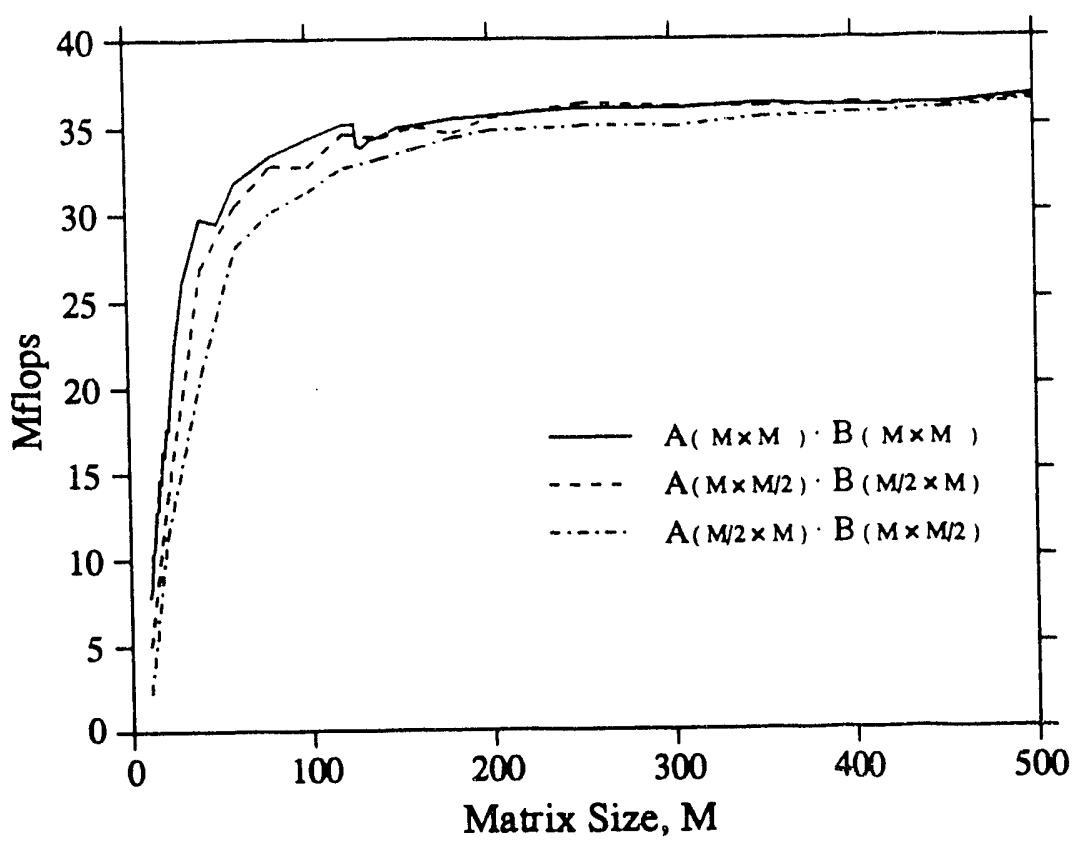

Figure 17: Performance of the assembly-coded Level 3 BLAS matrix multiplication routine DGEMM on one i 860 processor of the Intel Delta system. Results for square and rectangular matrices are shown. Note that the peak performance of about $35 \mathrm{Mflops}$ is attained only for matrices whose smallest dimension exceeds 100 . Thus, performance is improved if a few large matrices are multiplied by each process, rather than many small ones.

\subsection{Tradeoffs between Load Balance and Communication Latency}

We have discussed the mapping of the logical hierarchical memory to physical memory. In addition, we have pointed out the importance of maintaining long inner loops to get good sequential performance for each process, and the desirability of sending a few large messages rather than many smaller ones. We next consider load balance issues. Assuming that equal numbers of processes have been assigned to each processor, load imbalance arises in two phases of the parallel LU factorization algorithm; namely, in factoring each column block, which involves only $P$ processes, and in solving the lower triangular system to evaluate each row block of $U$, which involves only $Q$ processes. If the time for data movement is negligible, the aspect ratio of the template that minimizes load imbalance in step $k$ of the algorithm is,

$$
\begin{aligned}
\bar{P} & =\frac{\text { Sequential time to factor column block }}{\text { Sequential time for triangular solve }} \\
& =\frac{M_{b}-k-1 / 3+\mathrm{O}\left(1 / r^{2}\right)}{N_{b}-k-1+\mathrm{O}\left(1 / r^{2}\right)}
\end{aligned}
$$

where $M_{b} \times N_{b}$ is the matrix size in blocks, and $r$ the block size. Thus, the optimal aspect ratio of the template should be the same as the aspect ratio of the matrix, i.e., $M_{b} / N_{b}$ in 


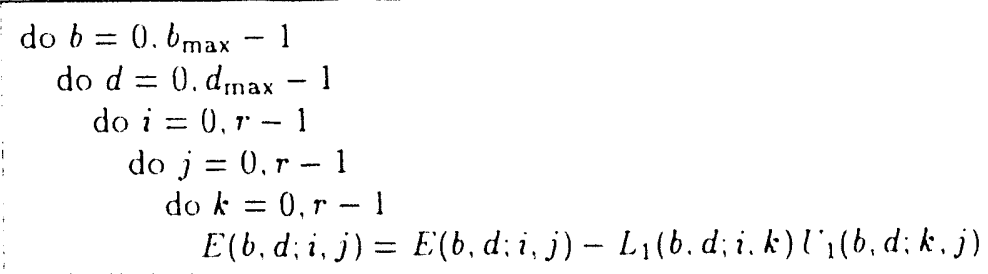

end all do loops

(a) Block-block multiplication

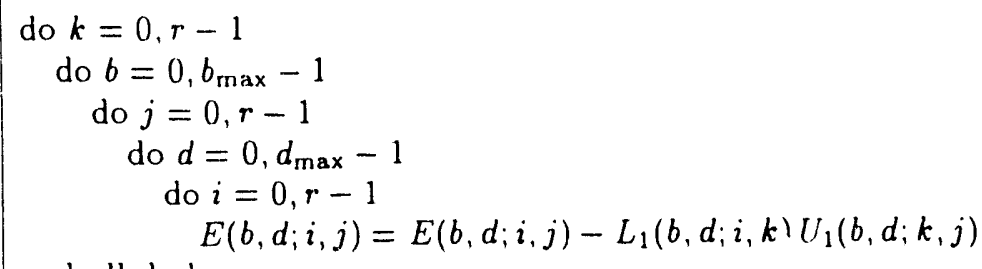

end all do loops

(b) Intermediate form of algorithm

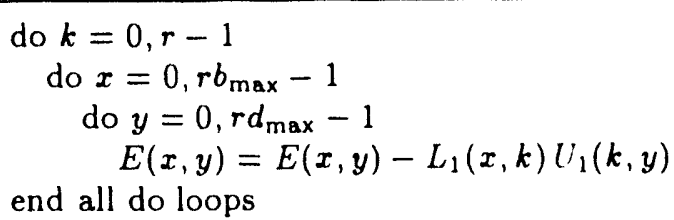

(c) Outer product form of algorithm

Figure 18: Pseudocode for different versions of the rank-r update, $E \leftarrow E-L_{1} U_{1}$, for one process. The number of row and column blocks per process is given by $b_{\max }$ and $d_{\max }$, respectively; $r$ is the block size. Blocks are indexed by $(b, d)$, and elements within a block by $(i, j)$. In version (a) the $r \times r$ blocks are multiplied one at a time, giving an inner loop of length $r$. (b) shows the loops rearranged before merging the $i$ and $d$ loops, and the $j$ and $b$ loops. This leads to the outer product form of the algorithm shown in (c) in which the inner loop is now of length $r d_{\max }$.

blocks, or $M / N$ in elements. If the effect of communication time is included then we must take into account the relative times taken to locate and broadcast the pivot information, and the time to broadcast the lower triangular matrix, $L_{0}$, along a row of the template. For both tasks the communication time increases with the number of processes involved, and since the communication time associated with the pivoting is greater than that associated with the triangular solve, we would expect the optimum aspect ratio of the template to be less than $M / N$ In fact, for our runs on the Intel Delta system we found an aspect ratio, $P / Q$, of between $1 / 4$ and $1 / 8$ to be optimal for most problems with square matrices, and that performance depends rather weakly on the aspect ratio, particularly for large grain sizes. Some typical results are shown in Figure 19 for 256 processors, which show a variation of less than $20 \%$ in performance 


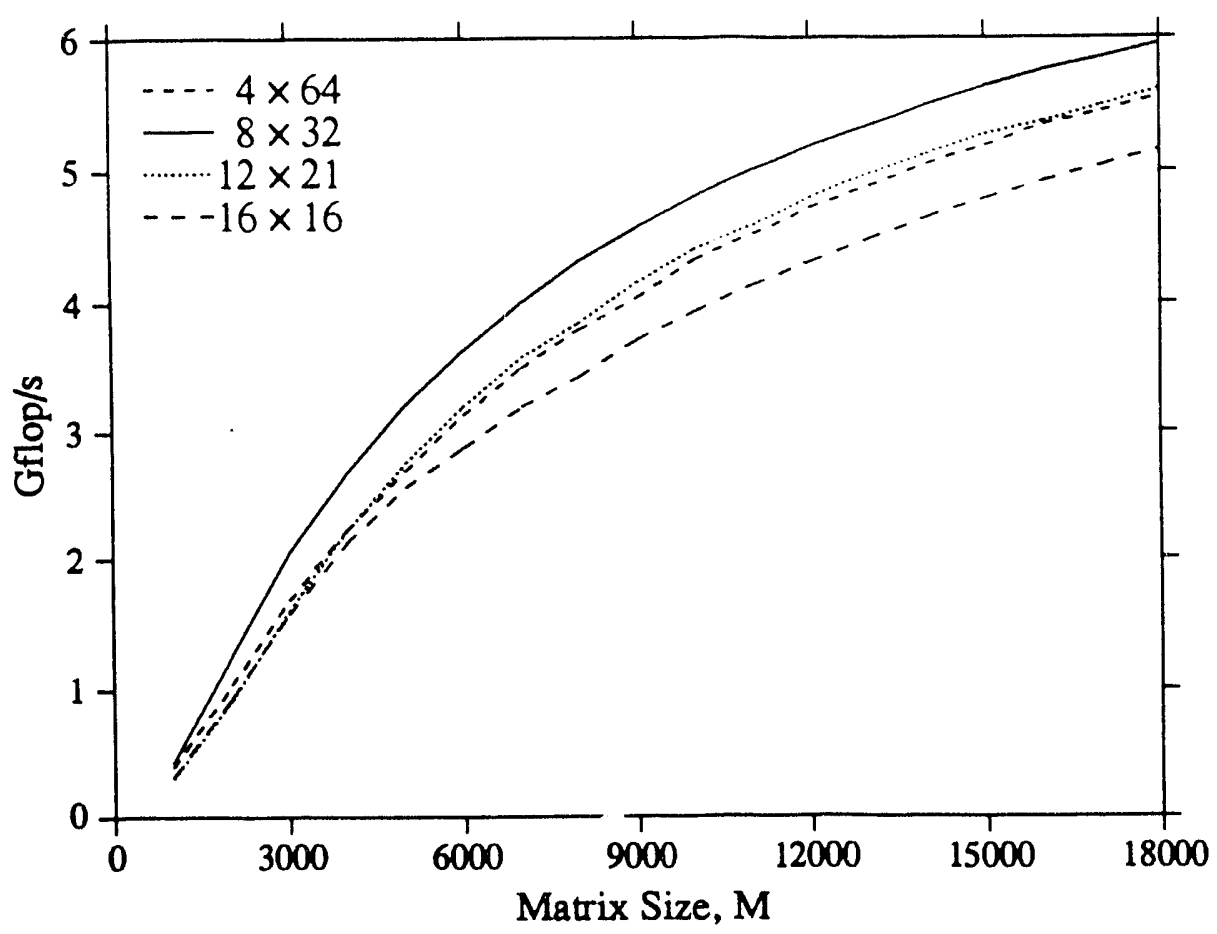

Figure 19: Performance of $\mathrm{LU}$ factorization on the Intel Delta as a function of square matrix size for different processor templates containing approximately 256 processors. The best performance is for an aspect ratio of $1 / 4$, though the dependence on aspect ratio is rather weak.

as $P / Q$ varies between $1 / 16$ and 1 for the largest problem.

The block size, $r$, also affects load balance. Here the tradeoff is between the load imbalance that arises as rows and columns of the matrix are eliminated as the algorithm progresses, and communication startup costs. The block cyclic decomposition seeks to maintain good load balance by cyclically assigning blocks to processes, and the load balance is best if the blocks are small. On the other hand, cumulative communication startup costs are less if the block size is large since, in this case, fewer messages must be sent (although the total volume of data sent is independent of the block size). Thus, there is a block size that optimally balances the load imbalance and communication startup costs.

\subsection{Optimality and Pipelining Tradeoffs}

The communication algorithms used also influence performance. In the $\mathrm{LU}$ factorization algorithm, all the communication can be done by moving data along rows and/or columns of the process template. This type of communication can be done by passing from one process to the next along the row or column. We shall call this a "ring" algorithm, although the ring 
may. or may not, be closed. An alternative is to use a spanning tree algorithm. of which there are several varieties. The complexity of the ring algorithm is linear in the number of processes involved, whereas that of spanning tree algorithms is logarithmic (for example, see [6]) Thus, considered in isolation. the spanning tree algorithms are preferable to a ring algorithm. However, in a spanning tree algorithm, a process may take part in several of the logarithmic steps, and in some implementations these algorithms act as a barrier. In a ring algorithm, each process needs to communicate only once, and can then continue to compute, in effect overlapping the communication with computation. An algorithm that interleaves communication and calculation in this way is often referred to as a pipelined algorithm. In a pipelined $\mathrm{LU}$ factorization algorithm with no pivoting, communication and calculation would flow in waves across the matrix. Pivoting tends to inhibit this advantage of pipelining.

In the pseudocode in Figure 15, we do not specify how the pivot information should be broadcast. In an optimized implementation, we need to finish with the pivot phase, and the triangular solve phase, as soon as possible in order to begin the update phase which is richest in parallelism. Thus, it is not a good idea to broadcast the pivot information from a single source process using a spanning tree algorithm, since this may occupy some of the processes involved in the panel factorization for too long. It is important to get the pivot information to the other processes in this template column as soon as possible, so the pivot information is first sent to these processes which subsequently broadcast it along the template rows to the other processes not involved in the panel factorization. In addition, the exchange of the parts of the pivot rows lying within the panel is done separately from that of the parts outside the pivot panel. Another factor to consider here is when the pivot information should be broadcast along the template columns. In Figure 15, the information is broadcast, and rows exchanged, immediately after the pivot is found. An alternative is to store up the sequence of $r$ pivots for a panel and to broadcast them along the template rows when panel factorization is complete. This defers the exchange of pivot rows for the parts outside the panel until the panel factorization has been done, as shown in the pseudocode fragment in Figure 20. An advantage of this second approach is that only one message is used to send the pivot information for the panel along the template rows, instead of $r$ messages.

In our implementation of LU factorization on the Intel Delta system, we used a spanning tree algorithm to locate the pivot and to broadcast it within the column of the process template performing the panel factorization. This ensures that pivoting, which involves only $P$ processes, is completed as quickly as possible. A ring broadcast is used to pipeline the pivot information and the factored panel along the template rows. Finally, after the triangular solve phase has completed, a spanning tree broadcast is used to send the newly-formed block row of $U$ along the template columns. Results for square matrices from runs on the Intel Delta system are shown 


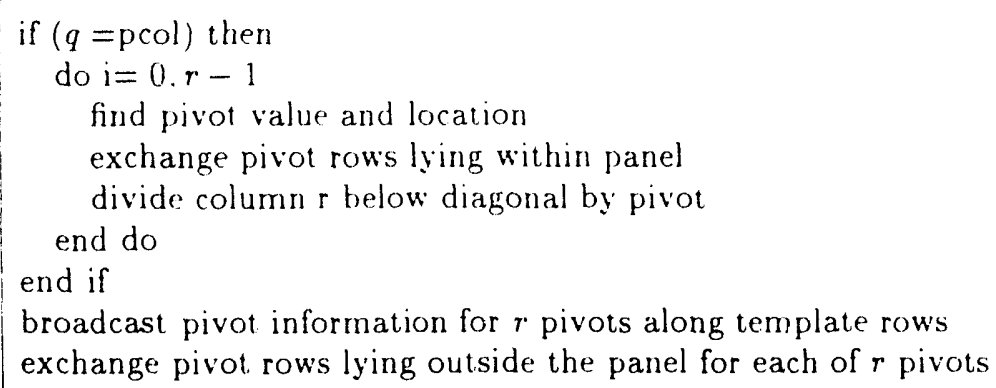

Figure 20: Pseudocode fragment for partial pivoting over rows. This may be regarded as replacing the first box inside the $k$ loop in Figure 15. In the above code pivot information is first disseminated within the template column doing the panel factorization. The pivoting of the parts of the rows lying outside the panel is deferred until the panel factorization has been completed.

in Figure 21. For each curve the results for the best process template configuration are shown. Recalling that for a scalable algorithm the performance should depend linearly on the number of processors for fixed granularity (see Eq. 2), it is apparent that scalability may be assessed by the extent to which sogranularity curves differ from linearity. An isogranularity curve is a plot of performance against number of processors for a fixed granularity. The results in Figure 21 can be used to generate the isogranularity curves shown in Figure 22 which show that on the Delta system the LU factorization routine starts to lose scalability when the granularity falls below about $0.2 \times 10^{6}$. This corresponds to a matrix size of about $M=10000$ on 512 processors, or about $13 \%$ of the memory available to applications on the Delta, indicating that LU factorization scales rather well on the Intel Delta system.

\section{Conclusions and Future Research Directions}

Portability of programs has always been an important consideration. Portability was easy to achieve when there was a single architectural paradigm (the serial von Neumann machine) and a single programming language for scientific programming (Fortran) embodying that common model of computation. Architectural and linguistic diversity have made portability much more difficult, but no less important, to attain. Users simply do not wish to invest significant amounts of time to create large-scale application codes for each new machine. Our answer is to develop portable software libraries that hide machine-specific details.

\subsection{Portability, Scalability, and Standards}

In order to be truly portable, parallel software libraries must be standardized. In a parallel computing environment in which the higher-level routines and/or abstractions are built 


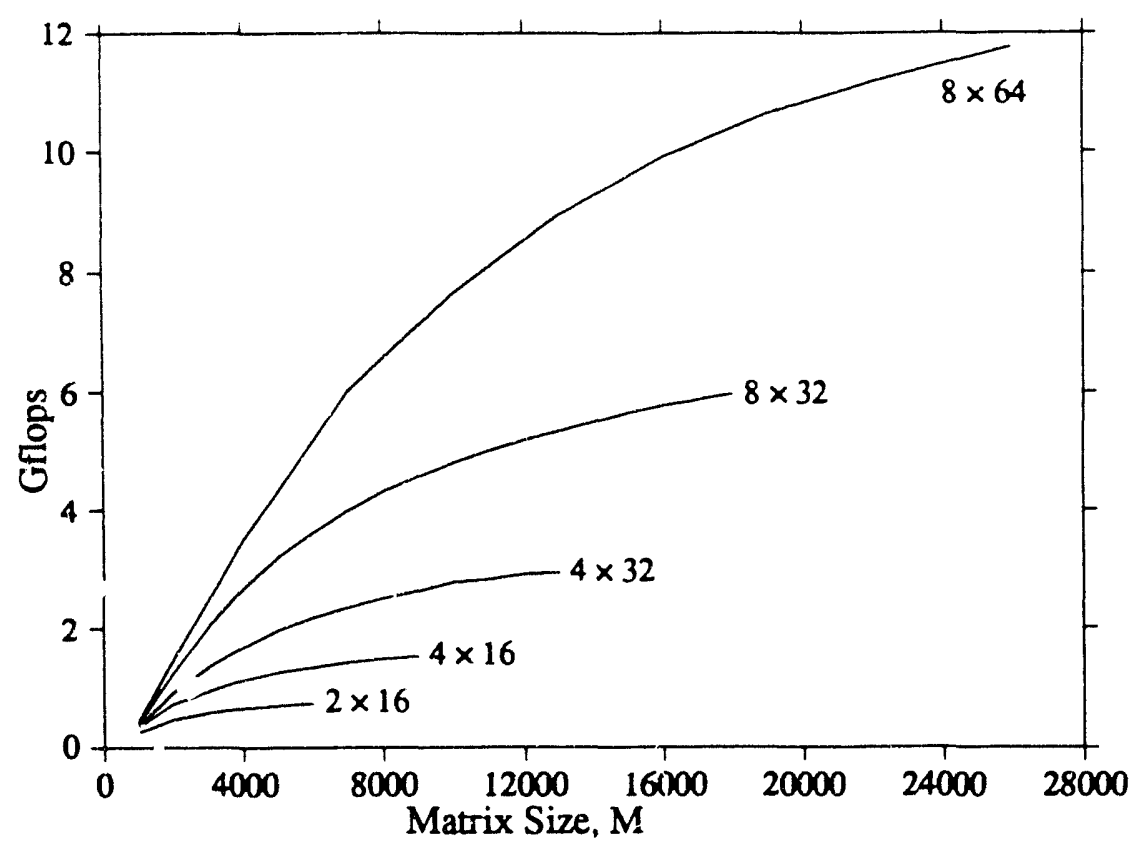

Figure 21: Performance of $L U$ factorization on the Intel Delta as a function of square matrix size for different numbers of processors. For each curve, results are shown for the process template configuration that gave the best performance for that number of processors.

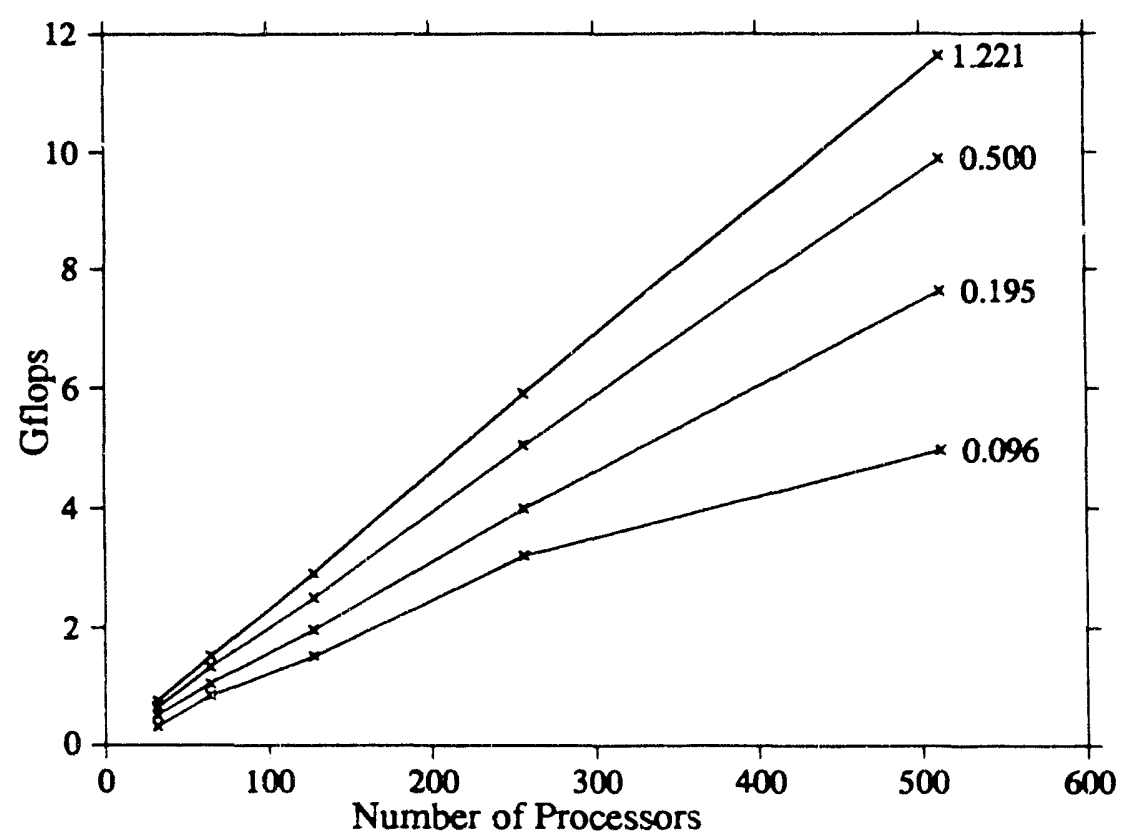

Figure 22: Isogranularity curves in the $\left(N_{p}, G\right)$ plane for the $\mathrm{LU}$ factorization of square matrices on the Intel Delta system. The curves are labeled by the granularity in units of $10^{6}$ matrix elements per processor. The linearity of the plots for granularities exceeding about $0.2 \times 10^{6}$ indicates that the LU factorization algorithm scales well on the Delta. 
upon lower-level computation and message-passing routines. the benefits of standardization are particularly apparent. Furthermore, the definition of computational and message-passing standards provides vendors with a clearly defined base set of routines that they can implement efficiently.

From the user's point of view, portability means that, as new machines are developed, they are simply added to the network, supplying cycles where they are most appropriate.

From the mathematical software developer's point of view, portability may require aignificant effort. Economy in development and maintenance of mathematical software demands that such development effort be leveraged over as many different computer systems as possible. Given the great diversity of parallel architectures, this type of portability is attainable to only a limited degree, but machine dependences can at least be isolated.

LAPACK is an example of a mathematical software package whose highest-level components are portable, while machine dependences are hidden in lower-level modules. Such a hierarchical approach is probably the closest one can come to software portability across diverse parallel architectures. And the BLAS that are used so heavily in LAPACK provide a portable, efficient, and flexible standard for applications programmers.

Like portability, scalability demands that a program be reasonably effective over a wide range of number of processors. The scalability of parallel algorithms, and software libraries based on them, over a wide range of architectural designs and numbers of processors will likely require that the fundamental granularity of computation be adjustable to suit the particular circumstances in which the software may happen to execute. Our approach to this problem is block algorithms with adjustable block size. In many cases, however, polyalgorithms ${ }^{1}$ may be required to deal with the full range of architectures and processor multiplicity likely to be available in the future.

Scalable parallel architectures of the future are likely $t$, be based on a distributed memory architectural paradigm. In the longer term, progress in hardware development, operating systems, languages, compilers, and communications may make it possible for users to view such distributed architectures (without significant loss of efficiency) as having a shared memory with a global address space. For the near term, however, the distributed nature of the underlying hardware will continue to be visible at the programming level; therefore, efficient procedures for explicit communication will continue to be necessary. Given this fact, standards for basic message passing (send/receive), as well as higher-level communication constructs (global summation, broadcast, etc.), become essential to the development of scalable libraries that have any degree of portability. In addition to standardizing general communication primitives, it

\footnotetext{
${ }^{I}$ In a polyalgorithm the actual algorithm used depenus on the computing environment and the input data. The optimal algorithm in a particular instance is altomaticall. selected at runtime.
} 
may also be advantageous to establish standards for problem-specific constructs in commonly occurring areas such as linear algebra.

The BLACS (Basic Linear Algebra Communication Subprograms) [16.26] is a package that provides the same ease of use and portability for MIMD message-passing linear algebra communication that the BLAS $[17,18,45]$ provide for linear algebra computation. Therefore, we recommend that future software for dense linear algebra on MIMD platforms consist of calls to the BLAS for computation and calls to the BLACS for communication. Since both packages will have been optimized for a particular platform, good performance should be achieved with relatively little effort. Also, since both packages will be available on a wide variety of machines, code modifications required to change platforms should be minimal.

\subsection{Alternative Approaches}

Traditionally, large, general-purpose mathematical software libraries have required users to write their own programs that call library routines to solve specific subproblems that arise during a computation. Adapted to a shared-memory parallel environment, this conventional interface still offers some potential for hiding underlying complexity. For example, the LAPACK project incorporates parallelism in the Level 3 BLAS, where it is not directly visible to the user.

But when going from shared-memory systems to the more readily scalable distributed memory systems, the complexity of the distributed data structures required is more difficult to hide from the user. Not only must the problem decomposition and data layout be specified, but different phases of the user's problem may require transformations between different distributed data structures.

These deficiencies in the conventional user interface have prompted extensive discussion of alternative approaches for scalable parallel software libraries of the future. Possibilities include:

1. Traditional function library (i.e., minimum possible change to the status quo in going from serial to parallel environment). This will allow one to protect the programming investment that has been made.

2. Reactive servers on the network. A user would be able to send a computational problem to a server that was specialized in dealing with the problem. This fits well with the concepts of a networked, heterogeneous computing environment with various specialized hardware resources (or even the heterogeneous partitioning of a single homogeneous parallel machine).

3. General interactive environments like Matlab or Mathematica, perhaps with "expert" drivers (i.e., knowledge-based systems). With the growing popularity of the many integrated packages based on this idea, this approach would provide an interactive, graphical 
interface for specifying and solving scientific problems. Both the algorithms and data structures are hidden from the user, because the package itself is responsible for storing and retrieving the problem data in an efficient. distributed manner. In a heterogeneous networked environment, such interfaces could provide seamless access to computational engines that would be invoked selectively for different parts of the user's computation according to which machine is most appropriate for a particular subproblem.

4. Domain-specific problem solving environments, such as those for structural analysis. En . vironments like Matlab and Mathematica have proven to be especially attractive for rapid prototyping of new algorithms and systems that may subsequently be implemented in a more customized manner for higher performance.

5. Reusable templates (i.e., users adapt "source code" to their particular applications). A template is a description of a general algorithm rather than the executable object code or the source code more commonly found in a conventional software library. Nevertheless, although templates are general descriptions of key data structures, they offer whatever degree of customization the user may desire.

Novel user interfaces that hide the complexity of scalable parallelism will require new concepts and mechanisms for representing scientific computational problems and for specifying how those problems relate to each other. Very high level languages and systems, perhaps graphically based, not only would facilitate the use of mathematical software from the user's point of view, but also would help to automate the determination of effective partitioning, mapping, granularity, data structures, etc. However, new concepts in problem specification and representation may also require new mathematical research on the analytic, algebraic, and topological properties of problems (e.g., existence and uniqueness).

We have already begun work on developing such templates for sparse matrix computations. Future work will focus on extending the use of templates to dense matrix computations.

We hope the insight we gained from our work will influence future developers of hardware, compilers and systems software so that they provide tools to facilitate development of high quality portable numerical software.

The EISPACK, LINPACK, and LAPACK linear algebra libraries are in the public domain, and are available from netlib. For example, for more information on how to obtain LAPACK, send the following one-line email message to netlibeornl.gov:

send index from lajack

Information for EISPACK and LINPACK can be similarly obtained. We expect to make a preliminary version of the ScaLAPACK library available from netlib in 1993. 


\section{Acknowledgments}

This research was performed in part using the Intel Touchstone Delta System operated by the California Institute of Technology on behalf of the Concurrent Supercomputing Consortium. Access to this facility was provided through the Center for Research on Parallel Computing.

\section{References}

[1] E. Anderson, A. Benzoni, J. J. Dongarra, S. Moulton, S. Ostrouchov, B. Tourancheau, and R. van de Geijn. LAPACK for distributed memory architectures: Progress report. In Parallel Processing for Scientific Computing, Fifth SIAM Conference. SIAM, 1991.

[2] E. Anderson and J. Dongarra. Results from the initial release of LAPACK. Technical Report LAPACK working note 16, Computer Science Department, University of 'Tennessee, Knoxville, TN, 1989.

[3] E. Anderson and J. Dongarra. Evaluating block algorithm variants in LAPACK. Technical Report LAPACK working note 19, Computer Science Department, University of Tennessee, Knoxville, TN, 1990.

[4] C. C. Ashcraft. The distributed solution of linear systems using the torus wrap data mapping. Engineering Computing and Analysis Technical Report ECA-TR-147, Boeing Computer Services, 1990.

[5] C. C. Ashcraft. A taxonamy of distributed dense LU factorization methods. Engineering Computing and Analysis Technical Report ECA-TR-161, Boeing Computer Services, 1991.

[6] M. Barnett, D. G. Payne, and R. yan de Geijn. Broadcasting on meshes with worm-hole routing. 'Technical report, Department of Computer Science, University of Texas at Austin, April 1993. Submitted to Supercomputing ' 93.

[7] W. S. Brainerd, C. H. Goldbergs, and J. C. Adams. Programmers Guide to Fortran 90. McGraw-Hill, New York, 1990.

[8] R. P. Brent. The LINPACK benchmark for the Fujitsu AP 1000. In Proceedings of the Fourth Symposium on the Frontiers of Massively Parallel Computation, pages 128-135. IEEE Computer Society Press, 1992.

[9] R. P. Brent. The LINPACK benchmark on the AP 1000: Preliminary report. In Proceedings of the 2nd CAP Workshop, NOV 1991. 
[10] J. Choi, J. J. Dongarra, R. Pozo, and D. W. Walker. Scalapack: A scalable linear algebra library for distributed memory concurrent computers. In Proceedings of the Fourth Symposium on the Frontzers of Massively Parallel Computation, pages 120-127. IEEE Computer Society Press, 1992.

[11] J. Choi, J. J. Dongarra, and D. W. Walker. The design of scalable software libraries for distributed memory concurrent computers. In J. J. Dongarra and B. Tourancheau, editors, Environments and Tools for Parallel Scientific Computing. Elsevier Science Publishers, 1993.

[12] E. Chu and A. George. Gaussian elimination with partial pivoting and load balancing on a multiprocessor. Parallel Computing, 5:65-74, 1987.

[13] D. E. Culler, A. Dusseau, S. C. Goldstein, A. Krishnamurthy, S. Lumetta, T. von Eicken, and K. Yelick. Introduction to Split-C: Version 0.9. Technical report, Computer Science Division - EECS, University of California, Berkeley, CA 94720, February 1993.

[14] J. Demmel. LAPACK: A portable linear algebra library for supercomputers. In Proceedings of the 1989 IEEE Control Systems Society Workshop on Computer-Aided Control System Design, December 1989.

[15] J. J. Dongarra. Increasing the performance of mathematical software through high-level modularity. In Proc. Sixth Int. Symp. Comp. Methods in Eng. \& Applied Sciences, Versailles, France, pages 239-248. North-Holland, 1984.

[16] J. J. Dongarra. LAPACK Working Note 34: Workshop on the BLACS. Computer Science Dept. Technical Report CS-91-134, University of Tennessee, Knoxville, TN, May 1991. (LAPACK Working Note \#34).

[17] J. J. Dongarra, J. Du Croz, S. Hammarling, and 1. Duff. A set of level 3 basic linear algebra subprograms. ACM Transactions on Mathematical Software, 16(1):1-17, 1990.

[18] J. J. Dongarra, J. Du Croz, S. Hammarling, and R. Hanson. An extended set of Fortran basic linear algebra subroutines. ACM Transactions on Mathematical Software, 14(1):117, March 1988.

[19] J. J. Dongarra, I. S. Duff, D. C. Sorensen, and H. A. Van der Vorst. Solving Linear Systems on Vector and Shared Memory Computers. SIAM Publications, Philadelphia, PA, 1991.

[20] J. J. Dongarra and E. Grosse. Distribution of mathematical software via electronic mail. Communications of the ACM, 30(5):403-407, July 1987. 
[21] J. J. Dongarra, R. Hempel, A. J. G. Hey, and D. W. Walker. A proposal for a userlevel message passing interface in a distributed memory environment. Technical Report TM-12231. Oak Ridge National Laboratory, February 1993.

[22] J. J. Dongarra, Peter Mayes, and Giuseppe Radicati di Brozolo. The IBM RISC System/6000 and linear algebra operations. Supercomputer, 44(V'III-4):15-30, 1991.

[23] J. J. Dongarra and S. Ostrouchov. LAPACK block factorization algorithms on the Intel iPSC/860. Technical Report CS-90-115, University of Tennessee at hnoxville, Computer Science Department, October 1990.

[24] J. J. Dongarra, R. Pozo, and D. W. Walker. An object oriented design for high performance linear algebra on distributed memory architectures. In Proceedings of the Object Oriented Numerics Conference, 1993.

[25] J. J. Dongarra, R. van de Geijn, and D. W. Walker. A look at scalable dense linear algebra libraries. In IEEE, editor, Proceedings of the Scalable High-Performance Computing Conference, pages 372-379. IEEE Publishers, 1992.

[26] J. J. Dongarra and R. A. van de Geijn. Two-dimensional basic linear algebra communication subprograms. Technical Report LAPACK working note 37, Computer Science Department, University of Tennessee, Knoxville, TN, October 1991.

[27] J. J. Dongarra and R. A. van de Geijn. Reduction to condensed form for the eigenvalue problem on distributed memory architectures. Parallel Computing, 18:973-982, 1992.

[28] J. Du Croz and M. Pont. The development of a floating-point validation package. In M. J. Irwin and R. Stefanelli, editors, Proceedings of the 8th Symposium on Computer Arithmetic, Como, Italy, May 19-21, 1987. IEEE Computer Society Press, 1987.

[29] T. H. Dunigan. Communication performance of the Intel Touchstone Delta mesh. Technical Report TM-11983, Oak Ridge National Laboratory, January 1992.

[30] A. Edelman. Large dense numerical linear algebra in 1993: The parallel computing influence. International Journal Supercomputer Applications, 1993. Accepted for publication.

[31] E. W. Felten and S. W. Otto. Coherent parallel C. In G. C. Fox, editor, Proceedings of the Third Conference on Hypercube Concurrent Computers and Applications, pages 440-450. ACM Press, 1988.

[32] G. C. Fox, M. A. Johnson, G. A. Lyzenga, S. W. Otto, J. K. Salmon, and D. W. Walker. Solving Problems on Concurrent Processors, volume 1. Prentice Hall, Englewood Cliffs, N.J., 1988. 
[33] K. Gallivan, R. Plemmons. and A. Sameh. Parallel algorithms for dense linear algebra computations. SI.AM Reveu, 32(1):54-135, 1990.

[34] A. Geist and M. Heath. Matrix factorization on a hypercube multiprocessor. In M. Heath, editor, Hypercube Multiprocessors. 1986, pages 161-180, Philadelphia, PA, 19866. Society for Industrial and Applied Mathematics.

[35] A. Geist and C. Romine. LU factorization algorithms on distributed-memory multiprocessor architectures. SIAM J. Sci. Statzst. Comput., 9(4):639-649, July 1988.

[36] G. H. Golub and C. F. Van Loan. Matrix Computations. The Johns Hopkins Press, Baltimore, Maryland, 2nd edition, 1989.

[37] A. Gupta and V. Kumar. On the scalability of FFT on parallel computers. In Proceedings of the Frontiers 90 Conference on Massively Parallel Computation. IEEE Computer Society Press, 1990. Also available as technical report TR 90-20 from the Computer Science Department, University of Minnesota, Minneapolis, MN 55455.

[38] R. Harrington. Origin and development of the method of moments for field computation. IEEE Antennas and Propagation Magazine, June 1990.

[39] B. Hendrickson and D. Womble. The torus-wrap mapping for dense matrix computations on massively parallel computers. Technical Report SAND92-0792, Sandia National Laboratories, April 1992.

[40] J. L. Hess. Panel methods in computational fluid dynamics. Annual Reviews of Fluid Mechanics, 22:255-274, 1990.

[41] J. L. Hess and M. O. Smith. Calculation of potential flows about arbitrary bodies. In D. Küchemann, editor, Progress in Aeronautical Sciences, Volume 8. Pergamon Press, 1967.

[42] High Performance Fortran Forum. High Performance Fortran Language Specification, Version 1.0, January 1993.

[43] R. W. Hockney and C. R. Jesshope. Parallel Computers. Adam Hilger Ltd., Bristol, UK, 1981.

[44] W. Kahan. Paranoia. Available from netlib [20].

[45] C. Lawson, R. Hanson, D. Kincaid, and F. Krogh. Basic linear algebra subprograms for Fortran usage. ACM Trans. Math. Sofiw., 5:308-323, 1979. 
[46] C. Leiserson. Fat trees: Universal networks for hardware-efficient supercomputing. IEEE Transactions on Computers, ('-34(10):892-901, 1985.

[47] W. Lichtenstein and S. L. Johnsson. Block-cyclic dense linear algebra. Technical Report TR-04-92, Harvard University; Center for Research in Computing Technology, January: 1992.

[48] M. Lin, D. Du, A. E. Klietz, and S. Saroff. Performance evaluation of the CM-5 interconnection network. Technical report, Department of Computer Science, University of Minnesota, 1992.

[49] R. Ponnusamy, A. Choudhary, and G. Fox. Communication overhead on CM-5: An experimental performance evaluation. In Proceedings of the Fourth Symposium on the Frontiers of Massively Parallel Computation, pages 108-115. IEEE Computer Society Press, 1992.

[50] Y. Saad and M. H. Schultz. Parallel direct methods for solving banded linear systems. Technical Report YALEU/DCS/RR-387, Department of Computer Science, Yale University, 1985 .

[51] S. R. Seidel. Broadcasting on linear arrays and meshes. Technical Report TM-12356, Oak Ridge National Laboratory, April 1993.

[52] A. Skjellum and A. Leung. LU factorization of sparse, unsymmetric, Jacobian matrices on multicomputers. In D. W. Walker and Q. F. Stout, editors, Proceedings of the Fifth Distributed Memory Concurrent Computing Conference, pages 328-337. IEEE Press, 1990.

[53] Thinking Machines Corporation, Cambridge, MA. CM-5 Technical Summary, 1991.

[54] R. A. van de Geijn. Massively parallel LINPACK benchmark on the Intel Touchstone Delta and iPSC/860 systems. Computer Science report TR-91-28, Univ. of Texas, 1991.

[55] E. F. Van de Velde. Data redistribution and concurrency. Parallel Computing, 16, December 1990 .

[56] J. J. H. Wang. Generalized Moment Methods in Electromagnetics. John Wiley \& Sons, New York, 1991.

[57] J. Wilkinson and C. Reinsch. Handbook for Automatic Computation: Volume II - Linear Algebra. Springer-Verlag, New York, 1971. 


\section{INTERNAL DISTRIBUTION}
1. B. R. Appleton
2. J. Choi
3-4. T. S. Darland
5. E. F. D'Azevedo
6-10. J. J. Dongarra
11. J. B. Drake
12. G. A. Geist
13. L. J. Gray
14. M. R. Leuze
15. E. G. Ng
16. C. E. Oliver
17. B. W. Peyton
18-22. S. A. Raby

\author{
23. C. H. Romine \\ 24. T. H. Rowan \\ 25-29. R. F. Sincovec \\ 30-34. D. W. Walker \\ 35-39. R. C. Ward \\ 40. P. H. Worley \\ 41. Central Research Library \\ 42. ORNL Patent Office \\ 43. K-25 Applied Technology Li- \\ brary \\ 44. Y-12 Technical Library \\ 45. Laboratory Records - RC. \\ 46-i7. Laboratory Records Department
}

\section{EXTERNAL DISTRIBUTION}

48. Cleve Ashcraft, Boeing Computer Services, P.O. Box 24346, M/S 7L-21, Seattle, WA 98124-0346

49. Donald M. Austin, 6196 EECS Bldg., University of Minnesota, 200 Union Street, S.E., Minneapolis, MN 55455

50. Robert G. Babb, Oregon Graduate Institute, CSE Department, 19600 N.W. von Neumann Drive, Beaverton, OR 97006-1999

51. Lawrence J. Baker, Exxon Production Research Company, P.O. Box 2189, Houston, TX 77252-2189

52. Jesse L. Barlow, Department of Computer Science, Pennsylvania State University, University Park, PA 16802

53. Edward H. Barsis, Computer Science and Mathematics, P. O. Box 5800, Sandia National Laboratories, Albuquerque, NM 87185

54. Michael L. Barton, Intel Corporation, 15201 N. W. Greenbrier Parkway, Beaverton, OR 97006

55. Colin Bennett, Department of Mathematics, University of South Carolina, Columbia, SC 29208

56. Dominique Bennett, CERFACS, 42 Avenue Gustave Coriolis, 31057 Toulouse Cedex, FRANCE

57. Marsha J. Berger, Courant Institute of Mathematical Sciences, 251 Mercer Street, New York, NY 10012 
58. Mike Berry, Department of Computer Science, Cniversity of Tennessee, 107 Ayres Hall. Knoxville. TN 37996-1301

59. Chris Bischof, Mathematics and Computer Science Division. Argonne National Laboratory. 9700 South Cass Avenue. Argonne. IL 60439

60. Ake Bjorck, Department of Mathematics. Linkoping University, S-581 83 Linkoping, Sweden

61-65. Jean R. S. Blair, Department of Computer Science, Ayres Hall, University of Tennessee, Knoxville, TN 37996-1301

66. Heather Booth, Department of Computer Science, Ayres Hall, University of Tennessee, Knoxville, TN 37996-1301

67. Roger W. Brockett, Harvard University, Pierce Hall, 29 Oxford Street, Cambridge, MA 02138

68. James C. Browne, Department of Computer Science, University of Texas, Austin, TX 78712

69. Bill L. Buzbee, Scientific Computing Division, National Center for Atmospheric Research, P.O. Box 3000, Boulder, CO 80307

70. Donald A. Calahan, Depas tment of Electrical and Computer Engineering, University of Michigan, Ann Arbor, MI 48109

71. John Cavallini, Acting Director, Scientific Computing Staff, Applied Mathematical Sciences, Office of Energy Research, U.S. Department of Energy, Washington, DC 20585

72. Ian Cavers, Department of Computer Science, University of British Columbia, Vancouver, British Columbia V6T 1W5, Canada

73. Tony Chan, Department of Mathematics, University of California, Los Angeles, 405 Hilgard A venue, Los Angeles, CA 90024

74. Jagdish Chandra, Army Research Office, P.O. Box 12211, Research Triangle Park, NC 27709

75. Eleanor Chu, Department of Computer Science, University of Waterloo, Waterloo, Ontario, Canada N2L 3G1

76. Melvyn Ciment, National Science Foundation, 1800 G Street N.W., Washington, DC 20550

77. Tom Coleman, Department of Computer Science, Cornell University, Ithaca, NY 14853

78. Paul Concus, Mathematics and Computing, Lawrence Berkeley Laboratory, Berkeley, CA 94720

79. Andy Conn, IBM T. J. Watson Research Center, P.O. Box 218, Yorktown Heights, NY 10598

80. John M. Conroy, Supercomputer Research Center, 17100 Science Drive, Bowie, MD 20715-4300 
81. Jane K. Cullum, IBM T. J. Watson Research Center. P.O. Box 218. Yorktown Heights. NY 10598

82. George Cybenko, Department of Math and Computer Science, Dartmouth College, Hanover, NH 03755

83. George J. Davis, Department of Mathematics. (ieorgia State University. Atlanta, GA 30303

84. Tim A. Davis, Computer and Information Sciences Department, 301 CSE, University of Florida, Gainesville, Florida 32611-2024

85. James Demmel, University of California, Computer Science Division, Berkeley, CA 94720

86. Frederic Desprez, Laboratoire de l'Information du Parallelisme, Ecole Normale Superieure de Lyon, 46, Allee d'Italie, 69364 Lyon Cedex 07, France

87. John J. Dorning, Department of Nuclear Engineering Physics, Thornton Hall, McCormick Road, University of Virginia, Charlottesville, VA 22901

88. Donald J. Dudziak, Department of Nuclear Engineering, 110B Burlington Engineering Labs, North Carolina State University, Raleigh, NC 27695-7909

89. Iain Duff, Numerical Analysis Group, Central Computing Department, Atlas Centre, Rutherford Appleton Laboratory, Didcot, Oxon OX11 0QX, England

90. Patricia Eberlein, Department of Computer Science, SUNY at Buffalo, Buffalo, NY 14260

91. Stanley Eisenstat, Department of Computer Science, Yale University, P.O. Box 2158 Yale Station, New Haven, CT 06520

92. Lars Elden, Department of Mathematics, Linkoping University, 58183 Linkoping, Sweden

93. Howard C. Elman, Computer Science Department, University of Maryland, College Park, MD 20742

94. Robert E. England, Mathematics and Computer Science Department, Northern Kentucky University, Highland Heights, KY 41076-1448

95. Albert M. Erisman, Boeing Computer Services, P.O. Box 24346, M/S 7L-21, Seattle, WA 98124-0346

96. Ian Foster, Mathematics and Computer Science Division, Argonne National Laboratory, 9700 South Cass Avenue, Argonne, IL 60439

97. Geoffrey C. Fox, Northeast Parallel Architectures Center, 111 College Place, Syracuse University, Syracuse, NY 13244-4100

98. Paul O. Frederickson, Center for Research on Parallel Computation, MS B287, Los Alamos National Laboratory, Los Alamos, NM 87545

99. Fred N. Fritsch, Computing \& Mathematics and Statistics Division, Lawrence Livermore National Laboratory, P.O. Box 808, L-316 Livermore, CA 94550 
100. Robert E. Funderlic. Department of Computer Science, North Carolina State Iniversity. Raleigh. NC 27650

101. K. Gallivan, Computer Science Department, University of Illinois, Urbana, IL 61801

102. Dennis B. Gannon. Computer Science Department. Indiana Eniversity, Bloomington. IN 47405

103. Feng Gao, Departmeni of Computer Science, University of British Columbia, Vancouver, British Columbia V6T 1W5, Canada

104. David M. Gay, Bell Laboratories, 600 Mountain Avenue, Murray Hill, NJ 07974

105. C. Williarn Gear, Computer Science Department, University of Illinois, Urbana, IL 61801

106. W. Morven Gentleman, Division of Electrical Engineering, National Research Council, Building M-50, Room 344, Montreal Road, Ottawa, Ontario, Canada K1A 0R8

107. J. Alan George, Vice President, Academic and Provost, Needles Hall, University of Waterloo, Waterloo, Ontario, Canada N2L 3G1

108. John R. Gilbert, Xerox Palo Alto Research Center, 3333 Coyote Hill Road, Palo Alto CA 94304

109. Ge ne H. Golub, Department of Computer Science, Stanford University, Stanford, CA 94305

110. Joseph F. Grcar, Division 8245, Sandia National Laboratories, Livermore, CA 94551-0969

111. John Gustafson, 236 Wilhelm, Ames Laboratory, Iowa State University, Ames, IA 50011

112. Per Christian Hansen, UCI*C Lyngby, Building 305, Technical University of Denmark, DK-2800 Lyngby, Denmark

113. Richard Hanson, IMSL Inc., 2500 Park West Tower One, 2500 City West Blvd., Houston, TX 77042-3020

114. Michael T. Heath, NCSA, University of Illinois, 4157 Beckman Institute, 405 North Mathews Avenue, Urbana, IL 61801-2300

115. Don E. Heller, Physics and Computer Science Department, Shell Development Co., P.O. Box 481, Houston, TX 77001

116. Anthony J. G. Hey, University of Southampton, Dept. Electronics and Comp. Sci., Highfield, Southampton SO9 5NH, United Kingdom

117. Nicholas J. Higham, Department of Mathematics, University of Manchester, Grt Manchester, M13 9PL, England

118. Charles J. Holland, Air Force Office of Scientific Research, Building 410, Bolling Air Force Base, Washington, DC 20332 
119. Fred Howes. Office of Scientific Computing ER- $\bar{T}$. Applied Mathematical Sciences. Office of Energy Research. L. S. Department of Energ. Washington. I) ( 2058i)

120. Robert E. Huddleston. Computation Department. Lawrence Livermore National Laboratory, P.O. Box 808, Livermore. CA 94550

121. John Huseby, Cray Research Superservers, Inc., $3601 \mathrm{~S}$. W. Murray Blvd.. Beaverton, OR 97005

122. Ilse Ipsen, Department of Computer Science, Yale University, P.O. Box 2158 Yale Station, New Haven, CT 06520

123. Elizabeth Jessup, University of Colorado, Department of Computer Science, Boulder, CO 80309-0430

124. Barry Joe, Department of Computer Science, University of Alberta, Edmonton, Alberta T6G 2H1. Canada

125. Gary Johnson, Office of Scientific Computing ER-7, Applied Mathematical Sciences, Office of Energy Research, U. S. Department of Energ, Washington, DC 20585

126. Lennart Johnsson, Thinking Machines Inc., 245 First Street, Cambridge, MA $02142-1214$

127. Harry Jordan, Department of Electrical and Computer Fngineering, University of Colorado, Boulder, CO 80309

128. Bo Kagstrom, Institute of Information Processing, University of Umea, 5-901 87 Umea, Sweden

129. David K. Kahaner, Office of Naval Research - Asia, Roppongi 7-23-17, Minato-ku, Tokyo 106, Japan

130. Malvyn H. Kalos, Cornell Theory Center, Engineering and Theory Center Bldg., Cornell University, Ithaca, NY 14853-3901

131. Hans Kaper, Mathematics and Computer Science Division, Argonne National Laboratory, 9700 South Cass Avenue, Bldg. 221, Argonne, IL 60439

132. Linda Kaufman, Bell Laboratories, 600 Mountain Avenue, Murray Hill, NJ 07974

133. Robert J. Kee, Division 8245, Sandia National Laboratories, Livermore, CA 945510969

134. Kenneth Kennedy, Department of Computer Science, Rice University, P.O. Box 1892, Houston, TX 77001

135. Eric S. Kirsch, Department of Computer Science, Ayres Hall, University of Tennessee, Knoxville, TN 37996-1301

136. Thomas Kitchens, Department of Energy, Scientific Computing Staff, Office of Energy Research, ER-7, Office G-236 Germantown, Washington, DC 20585

137. Michael A. Langston, Department of Computer Science, Ayres Hall, University of Tennessee, Knoxville, TN 37996-1301 
138. Richard Lau. Office of Naval Research. Code $1111 \mathrm{MA}, 800 \mathrm{~N}$. Quincy Street. Boston Tower 1 Arlington, VA 22217-5000

139. Alan J. Laub. Department of Electrical and Computer Engineering. University of California, Santa Barbara. CA 93106

140. Robert L. Launer, Army Research Office, P.O. Box 12211. Research Triangle Park. NC 27709

141. Charles Lawson. IriS 301-490. Jet Propulsion Laboratory, 4800 Oak Grove Drive, Pasadena, CA 91109

142. Peter D. Lax, Courant Institute of Mathematical Sciences, New York University, 251 Mercer Street, New York, NY 10012

143. James E. Leiss, Rt. 2, Box 142C, Broadway, VA 22815

144. John G. Lewis, Boeing Computer Services, P.O. Box 24346, M/S 7L-21, Seattle, WA $98124-0346$

145. Jing Li, IMSL Inc., 2500 Park West Tower One, 2500 City West Blvd., Houston, TX 77042-3020

146. Heather M. Liddell, Center for Parallel Computing, Department of Computer Science and Statistics, Queen Mary Westfield, University of London, Mile End Road, Lonc on El 4NS, England

147. Arno Liegm. nn, c/o ETH Rechenzentrum, Clausiusstr. 55, CH-8092 Zurich, Switzerland

148. Joseph Liu, Department of Computer Science, York University, 4700 Keele Street, North York, Ontario, Canada M3J 1P3

149. Robert F. Lucas, Supercomputer Research Center, 17100 Science Drive, Bowie, MD 20715-4300

150. Franklin Luk, Electrical Engineering Department, Cornell University, Ithaca, NY 14853

151. Brian A. Malloy, 216 Duke Street, Clemson, SC 296.3!

152. Thomas A. Manteuffel, Department of Mathematics, University of Colorado Denver, Campus Box 170. P.O. Box 173364, Denver, CO 80217-3364

153. John Martine, IBM Corporation. Dept. 85AA / Mail Station 276. Neighborhood Road, Kingston, NY 12401

154. James McGraw, Lawrence Livermore National Laboratory, L-306, P.O. Box 808, Livermore, CA 94550

155. Paul C. Messina, Mail Code 158-79. California Institute of Technology, 1201 E. California Blvd., Pasadena. CA 91125

156. Cleve Moler, The Mathworks, 24 Prime Park Way, Natick, MA 0176

157. Neville Moray, Department of Mechanical and Industrial Engineering, University of Illinois, 1206 West Green Street, Urbana, IL 61801 
158. Dr. David Nelson. Director of Scientific Computing. ER-T. Applied Mathematical Sciences. Office of Energy Research. C. S. Departnient of Energy, Washington. DC 20585

159. Dianne P. O'Leary, Compiter Science Department. University of Maryland. College Park, MD 20742

160. James M. Ortega, Departmeat of Applied Mathematics, Thornton Hall, University of Virginia, Charlottesville, VA 22901

161. Ct.arles F. Osgood, National Security Agency, Ft. George G. Meade, MD 20755

162. Steve Otto, Department of Computer Sci. \& Eng., Oregon Graduate Institute. 19600 N.W. von Neumann Drive, Beaverton, OR 97006-1999

163. ('hris Paige, OADDR, McGill University, McConnell Engineering Building 3480 University Street Montreal, PQ Canada H3A 2A 7

164. Roy P. Pargas, Department of Computer Science, Clemson University, Clemson, SC. 29634-1906

165. Beresford N. Parlett, Department of Mathernatics, University of California, Berkeley, CA 94720

166. Merrell Patrick, Department of Computer Science, Duke University, Durham, NC: 27706

167. Daniel J. Pierce, Boeing Computer Services, P.O. Box 24346, M/S 7L-21, Seattle, WA 98124-0346

168. Pobert J. Plemmons, Departments of Mathematics and Computer Science, Box 7311, Wake Forest University Winston-Salem, NC 27109

169. Jesse Poore, Department of Computer Science, Ayres Hall, University of Tennessee, Knoxville, TN 37996-1301

170. Alex Pothen, Department of Computer Science, Pennsylvania State University, University Park, PA 16802

171. Yuanchang Qi, IBM European Petroleum Application Center, P.O. Box 585, N4040 B̈:frsfjord, Norway

172. Giuseppe Radicati, IBM European Center for Scientific and Engineering Computing, via del Giorgione 159, I-00147 Roma, Italy

173. S. S. Ravi, Department of Computer Science, LI67A, 1400 Washington Avenue, Albany, NY 12222

174. John K. Reid, Numerical Analysis Group, Central Computing Department. Atlas Centre, Rutherford Appleton Laboratory, Didcot, Oxon OX 11 OQX, England

175. Werner C. Rheinboldt, Department of Mathematics and Statistics, University of Pittsburgh, Pittsburgh, PA 15260

176. John R. Rice, Computer Science Department, Purdue University, West Lafayette, IN 47907 
177. Garry Rodrigue, Numerical Mathematics Group, Lawrence Livermore Laboratory, Livermore, CA 94550

178. Donald J. Rose. Department of Computer Science, Duke University Durham, NC 27706

179-183. Bill Rosener, Department of Computer Science. Ayres Hall. Iniversity of 'Tennessee, Knoxville, TN 37996-1301

184. Edward Rothberg, Department of Computer Science, Stanford University, Stanford, CA 94305

185. Axel Ruhe, Department of Computer Science, Chalmers University of Technology, S-41296 Goteborg, Sweden

186. Joel Saltz, ICASE, MS 132C, NASA Langley Research Center, Hampton, VA 23665

187. Ahmed H. Sameh, Center for Supercomputing R\&D, 1384 W. Springfield Avenue, University of Illinois, Urbana, IL 61801

188. Michael Saunders, Systems Optimization Laboratory, Operations Research Department, Stanford University, Stanford, CA 94305

189. Robert Schreiber, RIACS, Mail Stop 230-5, NASA Ames Research Center, Moffet Field, CA 94035

190. Martin H. Schultz, Department of Computer Science, Yale University, P.O. Box 2158 Yale Station, New Haven, CT 06520

191. David S. Scott, Intel Scientific Computers, 15201 N.W. Greenbrier Parkway, Beaverton, OR 97006

192. Lawrence F. Shampine, Mathematics Department, Southern Methodist University, Dallas, TX 75275

193. Andy Sherman, Department of Computer Science, Yale University, P.O. Box 2158 Yale Station, New Haven, CT 06520

194. Kermit Sigmon, Department of Mathematics, University of Florida, Gainesville, FL 32611

195. Horst Simon, Mail Stop T045-1, NASA Ames Research Center, Moffett Field, CA 94035

196. Anthony Skjellum, Lawrence Livermore National Laboratory, 7000 East Ave., L316, P.O. Box 808 Livermore, CA 94551

197. Danny C. Sorensen, Department of Mathematical Sciences, Rice University, P. O. Box 1892, Houston, TX 77251

198. G. W. Stewart, Computer Science Department, University of Maryland, College Park, MD 20742

199. Paul N. Swartztrauber, National Center for Atmospheric Research, P.O. Box 3000, Boulder, CO 80307 
200. Michael G. Thomason, Department of Computer Science, Ayres Hall, Lniversity of Tennessee, Knoxville, TN 37996-1301

201. Philippe Toint, Department of Mathematics, Liniversity of Namur, FUNOP, 61 rue de Bruxelles, B-Namur, Belgium

202. Bernard Tourancheau, LIP, ENS-Lyon, 69364 Lyon cedex Oi, France

203. Anna Tsao, Supercomputing Research Center. 17100 Science Drive, Bowie, MD 20715

204. Robert van de Geijn, The University of Texas, Department of Computer Sciences. TAI 2.124, Austin, TX 78712

205. Hank Van der Vorst, Dept. of Techn. Mathematics and Comp. Sci., Delft University of Technology, P.O. Box 356, NL-2600 AJ Delft, The Netherlands

206. Charles Van Loan, Department of Computer Science, Cornell University, Ithaca, NY 14853

207. Jim M. Varah, Centre for Integrated Computer Systems Research, University of British Columbia, Office 2053-2324 Main Mall, Vancouver, British Columbia V6T 1W5, Canada

208. Udaya B. Vemulapati, Department of Computer Science, University of Central Florida, Orlando, FL 32816-0362

209. Robert G. Voigt, National Science Foundation, Room 417, 1800 G Street, N.W., Washington, DC 20550

210. Phuong Vu, Cray Research, Inc., 1345 Northland Dr., Mendota Heights, MN 55120

211. Kevin Wadleigh, Convex Computer Corporation, 3000 Waterview Parkway, P. O. Box 833851, M.S. MAR, Richardson, TX 75083-3851

212. Daniel D. Warner, Department of Mathematical Sciences, O-104 Martin Hall. Clemson University, Clemson, SC 29631

213. Gilbert G. Weigand, Computing Systems Technology Office, Advanced Research Projects Agency, 3701 North Fairfax Drive, Arlington, VA 22203-1714

214. Mary F. Wheeler, Rice University, Department of Mathematical Sciences, P.O. Box 1892, Houston, TX 77251

215. Andrew B. White, Computing Division, Los Alamos National Laboratory, P.O. Box 1663, MS-265, Los Alamos, NM 87545

216. Michael Wolfe, Department of Computer Sci. \& Eng., Oregon Graduate Institute, 19600 N.W. von Neumann Drive, Beaverton, OR 97006-1999

217. David Womble, Sandia National Laboratories, Numerical Mathernatics Division 1422, P. O. Box 5800 Albuquerque, NM 87185

218. Margaret Wright, Bell Laboratories, 600 Mountain Avenue, Murray Hill, NJ 07974 
219. David Young, University of Texas. Center for Numerical Analysis, RLM 13.150. Austin, $\mathrm{TX} 78731$

220. Earl Zmijewski, Department of Computer Science, University of California, Santa Barbara, CA 93106

221. Office of Assistant Manager for Energy Research and Development, U.S. Department of Energy, Oak Ridge Operations Office, P.O. Box 2001 Oak Ridge, TN 37831-8600

222-223. Office of Scientific \& Technical Information, P.O. Box 62, Oak Ridge, TN 37830 

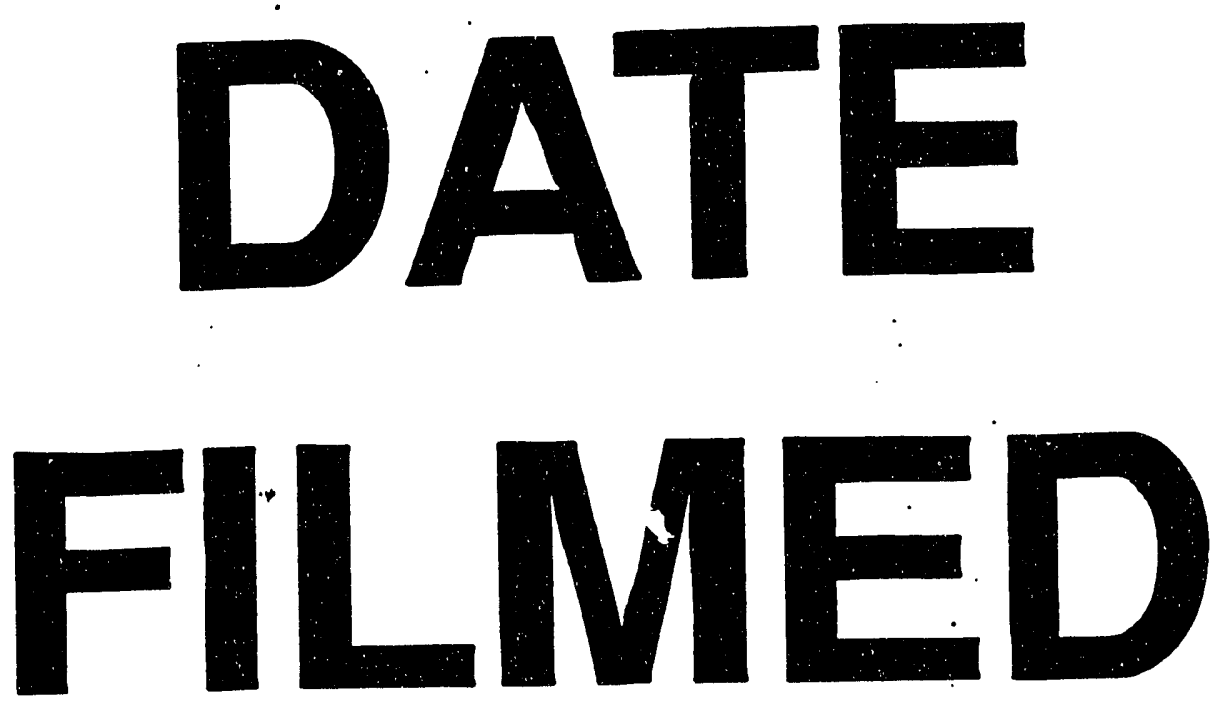

$11 / 26 / 93$
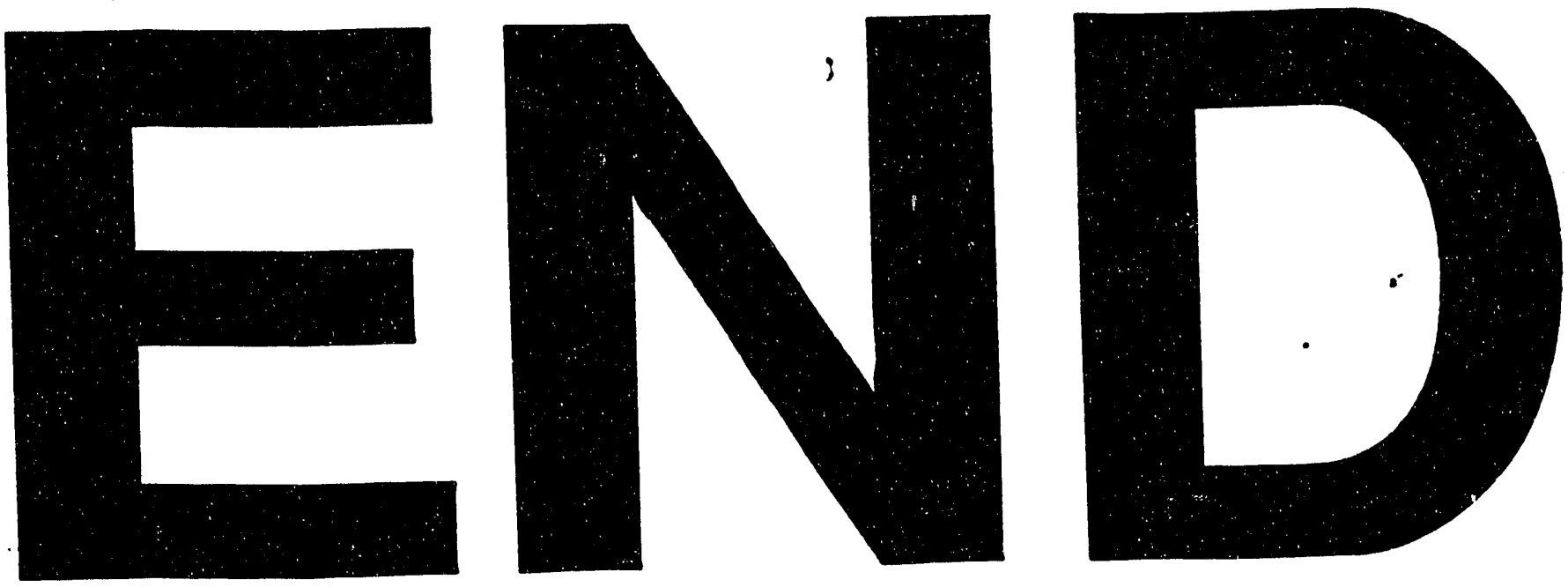
